\title{
A beamspace approach for 2-D localization of incoherently distributed sources in massive MIMO systems
}

\author{
Tiejun Lv ${ }^{a}$, Fangqing Tan ${ }^{a}$, Hui Gao ${ }^{a, *}$, Shaoshi Yang ${ }^{b}$ \\ a School of Information and Communication Engineering, Beijing University of Posts and Telecommunications, Beijing 100876, China \\ ${ }^{\mathrm{b}}$ School of Electronics and Computer Science, University of Southampton, SO17 1BJ Southampton, UK
}

\section{A R T I C L E I N F O}

\section{Article history:}

Received 20 June 2015

Received in revised form

20 September 2015

Accepted 21 October 2015

Available online 30 October 2015

\section{Keywords:}

Beamspace

Incoherently distributed (ID) source Large scale/massive multiple-input multiple-output systems (LS-MIMO/massive MIMO)

Multiple-input multiple-output (MIMO)

Uniform cylindrical array (UCyA)

\begin{abstract}
A B S T R A C T
In this paper, a generalized low-complexity beamspace approach is proposed for twodimensional localization of incoherently distributed sources with a uniform cylindrical array (UCyA) in large scale/massive multiple-input multiple-output (MIMO) systems. The received signal vectors in the antenna-element space are transformed into the beamspace by employing beamforming vectors. As a beneficial result, the total dimensions of the received signal vectors are significantly reduced. In addition, it is shown that the error introduced by the transformation decreases as the number of UCyA antennas increases. The UCyA is composed of multiple uniform circular arrays (UCAs), and the beamspace array response matrices of adjacent UCAs are linearly related. Then, the linear relation is exploited to estimate the nominal elevation direction-of-arrivals (DOAs) directly and the nominal azimuth DOAs based on a lowcomplexity search algorithm. In contrast, the linear relation in the traditional approach is based on approximations and the associated search algorithm is more complicated. Numerical results demonstrate that the proposed approach outperforms the existing approach in terms of both performance and complexity in the context of massive MIMO systems.
\end{abstract}

(c) 2015 Elsevier B.V. All rights reserved.

\section{Introduction}

Multiple-input multiple-output (MIMO) techniques are capable of providing a flexible tradeoff between multiplexing gain and diversity gain, which may significantly improve both the spectral efficiency and the link reliability of wireless communication systems [1]. Recently, massive MIMO, which is also called large-scale MIMO, has been shown to achieve extremely high spectral efficiency [2,3]. In massive MIMO systems, the base station (BS) is equipped with a hundred or a few hundred antennas, serving tens of user terminals (UTs) simultaneously. However, the performance of these systems degrades when the angular

\footnotetext{
* Corresponding author.

E-mail addresses: lvtiejun@bupt.edu.cn (T. Lv), tfqing@bupt.edu.cn (F. Tan), huigao@bupt.edu.cn (H. Gao), sy7g09@ecs.soton.ac.uk (S. Yang).
}

spreads are not wide enough, hence a beamforming approach is proposed to achieve directional antenna gain [4]. Meanwhile, the large number of antennas have become feasible due to the employment of multidimensional antenna arrays, such as uniform rectangular array (URA) and uniform cylindrical array (UCyA). As a result, the beamforming might be implemented not only in the azimuth direction, but also in the elevation direction [2]. This is known as the three-dimensional (3-D) beamforming $[5,6]$ that can improve spectral efficiency. The 3-D beamforming requires the position knowledge of the UTs, which corresponds to the two-dimensional (2-D), i.e., angular parameters, azimuth and elevation. Therefore, 2-D localization for 3-D beamforming in massive MIMO systems, which is the focus of this paper, constitutes an important problem.

The localization of point sources has been extensively investigated till now [7]. The point source model is 
suitable for line-of-sight transmission scenario, in which the signal of each source impinges on the array from a single angle [8]. When the signal of one source arrives from an angular region, the source is modeled as a distributed source, which is suitable for the multipath transmission scenario [9]. Moreover, the distributed sources may be categorized into coherently distributed (CD) and incoherently distributed (ID) sources [10], which are appropriate for slowly time-varying and rapidly timevarying channels, respectively. The cellular wireless channels are usually rapidly time-varying, thus the UTs of cellular wireless systems are regarded as ID sources.

The localization of $C D$ sources has been tackled by the classical approaches conceived for point sources [8,10-12], while the localization of ID sources is generally more challenging [13-34]. Although most of the existing ID source localization methods are proposed for onedimensional (1-D) scenarios, where only the azimuth parameters have to be estimated, some of them can be extended to 2-D scenarios. Among the existing approaches for 2-D localization of ID sources, the maximum likelihood (ML) approach [17], the approximate-ML approach [18], and the least-squares (LS)-based covariance matching approach [17,26,27] can achieve optimal or near optimal performance. However, the search dimensions of these methods are too high for practical implementation, especially in the context of massive MIMO systems.

The high computational complexity is the main problem for 2-D localization of ID sources in massive MIMO systems, for which the existing approaches are not suitable. By introducing a simplified signal model, the search dimensions of the above-mentioned approximate-ML and LS-based approaches may be significantly reduced [15,20-25], but these modified approaches are limited to the single-source case. On the other hand, by generalizing the multiple signal classification (MUSIC) [35] and beamforming approaches to ID sources [28-32], the computational complexity can be reduced in comparison with the ML-based [17] and the LSbased approaches $[17,26,27]$. However, the search of the 2-D nominal direction-of-arrivals (DOAs) and angular spreads still results in high computational complexity. Meanwhile, the estimation of signal parameters via rotational invariance technique (ESPRIT) [36-38] has also been generalized for estimating the 2-D nominal DOAs with two uniform circular arrays (UCAs), and only the nominal azimuth DOAs have to be estimated by searching [34]. Hence, the computational complexity of this approach is lower than that of the abovementioned other methods [17,18,26-32]. Unfortunately, the computational complexity of the ESPRIT based approach [34] remains proportional to the cubic of the number of BS antennas. Additionally, the approximations for deriving the linear relation in this approach are in fact unnecessary. The ESPRIT approach has also been extended to estimate both the 2-D nominal DOAs and the angular spreads of ID sources in massive MIMO systems, imposing a low computational complexity [39], but this approach can only be employed with URAs. Furthermore, in contrast to the above-mentioned methods that are all based on the antenna-element space, the beamspace based approach was shown to be capable of achieving low computational complexity [40], hence it has been employed to improve the conventional element-spaced based ESPRIT in $[41,42]$. However, these beamspace ESPRIT approaches were limited to point sources in small- or medium-dimension systems. Therefore, low-complexity 2-D localization algorithms need to be investigated for ID sources in the context of massive MIMO systems.

In this paper, a generalized beamspace approach is proposed for 2-D localization of ID sources in the context of massive MIMO systems that rely on a large UCyA composed of multiple UCAs. It is worth emphasizing that while the traditional beamspace approaches in [40-42] were conceived for point sources in small- or medium-dimension systems, and it is well known that the localization of point sources is quite different from that of ID sources. We transform the received signal vectors in the element space to the beamspace by employing beamforming vectors, and this is known as the beamspace transform [43]. Then, the linear relation between the beamspace array response matrices of the UCAs is exploited to estimate nominal elevation DOAs. Subsequently, the nominal azimuth DOAs are estimated by performing 1-D search. Finally, the 2-D angular spreads can be estimated with the aid of these previous attained estimates. For the purpose of clarity, the main contributions of this paper are summarized as follows:

(1) A beamspace approach with significantly lower computational complexity than the traditional element space method [34] is proposed. The dimensions of the received signal vectors are significantly reduced after the beamspace transform. As a result, the computational complexity of the proposed approach is linearly proportional to the number of BS antennas. Moreover, it is shown that the error introduced by the beamspace transform tends to zero as the number of BS antennas tends to infinity.

(2) A new search criterion for estimating nominal azimuth DOAs is conceived. Consequently, the performance of the proposed approach is better than that of [34], and is closed to the approximate Cramér-Rao bound (CRB).

(3) We prove that the approximations in [34] are not necessary. The linear relation between the element space array response matrices of adjacent UCAs is derived without the approximations in [34].

This paper is organized as follows. In Section 2, a detailed description of the system is presented. The beamspace transform for large UCAs is derived in Section 3. In Section 4, the derivation of the proposed channel estimator is expounded. The theoretical analysis regarding the proposed approach is presented in Section 5. In Section 6 , simulation results and further discussions are provided. Finally, conclusions are drawn in Section 7.

Notations: Lower-case (upper-case) boldface symbols denote vectors (matrices); $\mathbf{I}_{K}$ represents the $K \times K$ identity matrix, and $\mathbf{0}_{M \times K}$ represents an $M \times K$ zero matrix; $\operatorname{diag}(\cdot)$ denotes a diagonal matrix and the values in the parentheses constitute its diagonal elements; $(\cdot)^{*},(\cdot)^{T},(\cdot)^{H},(\cdot)^{\dagger}$, and $\mathbb{E}\{\cdot\}$ denote the conjugate, the transpose, the conjugate transpose, the pseudoinverse, and the expectation, respectively; $[\cdot]_{j, k}$ and $\|\cdot\|_{F}$ represent the $(j, k)$ th entry and the Frobenius norm of a matrix, respectively; $\odot$ is the Hadamard product operator; []$_{j}$ is the $j$ th element of a vector; \lceil\rceil gives the 


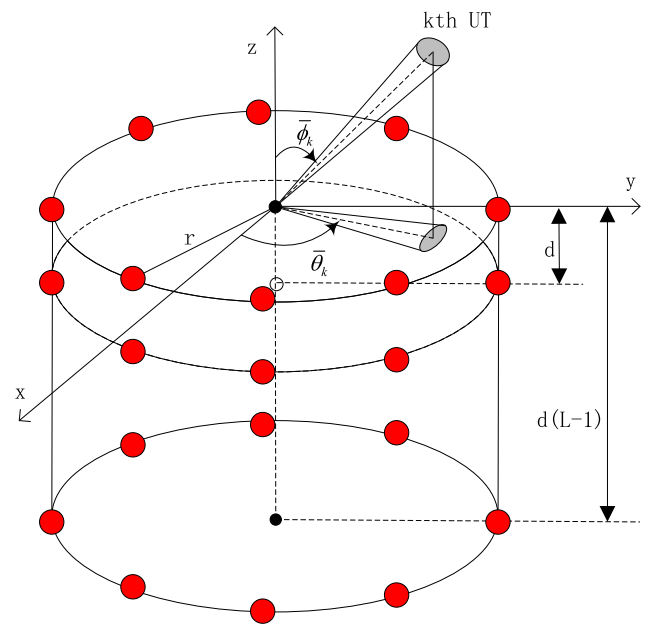

Fig. 1. The UCyA geometry considered. The array is composed of $L$ vertically aligned and concentric UCAs. Each UCA is composed of $N$ elements, and the total number of elements is $M=N L$. The radius of each UCA is $r$, and the vertical spacing between adjacent UCAs is $d$. The nominal azimuth DOA and elevation DOA of the $k$ th UT are $\bar{\theta}_{k}$ and $\bar{\phi}_{k}$, respectively.

smallest integer that is greater than the value; $i$ is the imaginary unit; finally, $\delta(\cdot)$ denotes the Kronecker delta function.

\section{System description}

The geometry of the UCyA at the BS is depicted in Fig. 1. The array is composed of $L$ vertically aligned and concentric UCAs. Each UCA is composed of $N$ antenna elements, and the total number of antenna elements is $M=N L$. The radius of each UCA is $r$, and the vertical spacing between any two adjacent UCAs is $d$. In this case, the height of the UCyA is $d(L-1)$. The UCA at the top lies on the $x y$-plane, and the center of this UCA is also the origin of the coordinates. The vertical axis of the UCyA is aligned with the $z$-axis. The elevation angle is measured downward from the $z$-axis, and the azimuth angle is measured counterclockwise from the $x$-axis. Additionally, the geometry of the UCA at the top is depicted in Fig. 2. As shown in both Figs. 1 and 2, the $N$ antennas of a given UCA are uniformly distributed over the circumference of a circle of a radius $r$. Hence, the position of the $n$th antenna element of a given UCA is represented by the angle $\vartheta_{n}=2 \pi(n-1) / N, n=1,2, \ldots, N$, which is measured counterclockwise from the $x$-axis. The antenna elements of the other UCAs are positioned in the same manner, so that the $M$ antenna elements constitute $N$ vertical columns, and each column consists of $L$ antenna elements.

We consider that there are $K$ single-antenna UTs transmitting signals to the BS, and these signals propagate through multipaths before impinging on the UCyA. As shown in Fig. 1, it is assumed that the reflectors are near to the UTs. Thus, the signals received at the BS from the $k$ th UT can be regarded as a spatially distributed cluster, and the UTs can be regarded as distributed sources. At the tth time instant, the received signal vector at the UCyA is modeled as

$\mathbf{x}(t)=\sum_{k=1}^{K} s_{k}(t) \sum_{j=1}^{N_{k}} \gamma_{k, j}(t) \mathbf{a}\left(\theta_{k, j}(t), \phi_{k, j}(t)\right)+\mathbf{n}(t) \in \mathbb{C}^{M \times 1}$,

where $s_{k}(t)$ is the signal from the $k$ th UT; $N_{k}$ is the number of multipaths associated with the $k$ th UT; $\gamma_{k, j}(t), \theta_{k, j}(t)$, and $\phi_{k, j}(t)$ are the complex-valued gain, the azimuth DOA and the elevation DOA of the $j$ th multipath of the signal sent from the $k$ th UT, respectively; $\mathbf{n}(t) \in \mathbb{C}^{M \times 1}$ is the complexvalued received noise vector. Additionally, $\mathbf{a}\left(\theta_{k, j}(t), \phi_{k, j}(t)\right) \in$ $\mathbb{C}^{M \times 1}$ is the array steering vector having the form

$$
\begin{aligned}
& {\left[\mathbf{a}\left(\theta_{k, j}(t), \phi_{k, j}(t)\right)\right]_{m}=\exp \left(i \mu \left[r \sin \left(\phi_{k, j}(t)\right) \times \cos \left(\theta_{k, j}(t)-\vartheta_{n}\right)\right.\right.} \\
& \left.\left.\quad-d(l-1) \cos \left(\phi_{k, j}(t)\right)\right]\right)
\end{aligned}
$$

where $m=N(l-1)+n, n=1,2, \ldots, N, l=1,2, \ldots, L, \quad \mu=2 \pi$ $/ \lambda$ is the wavenumber, and $\lambda$ is the wavelength. Note that in the definition of the array steering vector $\mathbf{a}\left(\theta_{k, j}(t), \phi_{k, j}(t)\right)$, the received signal phase ${ }^{1}$ at the origin of the coordinates is taken as the reference phase. The ranges of the azimuth DOA $\theta_{k, j}(t)$ and elevation DOA $\phi_{k, j}(t)$ are $0 \leq \theta_{k, j}(t)<2 \pi$ and $0 \leq \phi_{k, j}(t) \leq \pi / 2$, respectively, which means that the UCyA can provide $360^{\circ}$ azimuth coverage and $90^{\circ}$ elevation coverage. Though the UCyA can provide $180^{\circ}$ elevation coverage in practice, the array is placed above the UTs, and the range of elevation is only $0^{\circ}$ to $90^{\circ}$. Hence, we assume that the range of the elevation DOA is $0 \leq \phi_{k, j}(t) \leq \pi / 2$. The azimuth DOA $\theta_{k, j}(t)$ and elevation DOA $\phi_{k, j}(t)$ are expressed as [27]

$\theta_{k, j}(t)=\bar{\theta}_{k}+\tilde{\theta}_{k, j}(t)$

and

$\phi_{k, j}(t)=\bar{\phi}_{k}+\tilde{\phi}_{k, j}(t)$,

respectively, where $\bar{\theta}_{k}$ and $\bar{\phi}_{k}$ are the means of $\theta_{k, j}(t)$ and $\phi_{k, j}(t)$, respectively, i.e., they are the nominal azimuth DOA and nominal elevation DOA of the $k$ th UT, as shown in Fig. 1 ; additionally, the angular deviations $\tilde{\theta}_{k, j}(t)$ and $\tilde{\phi}_{k, j}(t)$ are zero-mean random variables with variances $\sigma_{\theta_{k}}^{2}$ and $\sigma_{\phi_{k}}^{2}$, respectively. To elaborate a little further, $\sigma_{\theta_{k}}$ and $\sigma_{\phi_{k}}$ are known as the azimuth and elevation angular spreads, respectively. It should be noted that the task of 2-D localization is to estimate the angular parameters $\bar{\theta}_{k}, \bar{\phi}_{k}, \sigma_{\theta_{k}}$, and $\sigma_{\phi_{k}}, k=1,2, \ldots, K$, with the aid of the received signal snapshots $\mathbf{x}(t), t=1,2, \ldots, T$, where $T$ is the number of received signal snapshots. Then, the estimated 2-D angular parameters can be used in the 3-D beamforming, which is capable of increasing the signal-tointerference ratios of the UTs.This paper focuses on the 2-D localization of multiple ID sources.

In this paper, the following initial assumptions are considered.

(1) The angular deviations, $\tilde{\theta}_{k, j}(t)$ and $\tilde{\phi}_{k, j}(t), k=$ $1,2, \ldots, K, \quad j=1,2, \ldots, N_{k}, \quad t=1,2, \ldots, T$, are temporally

\footnotetext{
${ }^{1}$ For the azimuth angle $\theta_{k, j}(t)$ and elevation angle $\phi_{k, j}(t)$, the difference between the received signal phase at the $m$ th antenna and the received signal phase at the origin is $\mu\left[r \sin \left(\phi_{k, j}(t)\right) \cos \right.$ $\left.\left(\theta_{k, j}(t)-\vartheta_{n}\right)-d(l-1) \cos \left(\phi_{k, j}(t)\right)\right]$, cf. (2).
} 


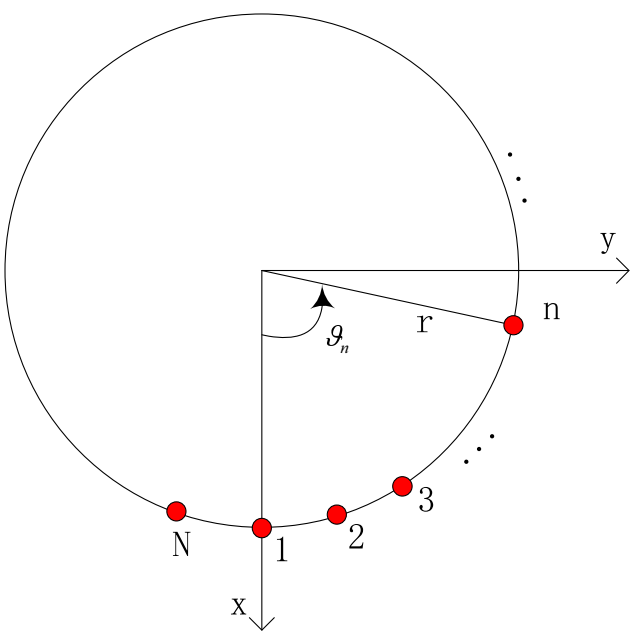

Fig. 2. The constituent UCA geometry considered. The array is composed of $N$ elements, and the radius of the UCA is $r$. The $n$th element of the UCA is positioned at an angle $\vartheta_{n}=2 \pi(n-1) / N, n=1,2, \ldots, N$ which is measured by turning counterclockwise from the $x$-axis.

independent and identically distributed (i.i.d.) Gaussian random variables with covariances

$\mathbb{E}\left\{\tilde{\theta}_{k, j}(t) \tilde{\theta}_{\tilde{k}, \tilde{j}}(\tilde{t})\right\}=\sigma_{\theta_{k}}^{2} \delta(k-\tilde{k}) \delta(j-\tilde{j}) \delta(t-\tilde{t})$

and

$\mathbb{E}\left\{\tilde{\phi}_{k, j}(t) \tilde{\phi}_{\tilde{k}_{i} \tilde{j}}(\tilde{t})\right\}=\sigma_{\phi_{k}}^{2} \delta(k-\tilde{k}) \delta(j-\tilde{j}) \delta(t-\tilde{t})$,

respectively. Note that the Gaussian distribution is assumed [49] for the sake of simplicity in the subsequent analysis. In fact, we can change this assumption and use the Uniform, the Laplacian or the Von-Mises distributions, since the proposed approach is independent of the specific distributions of the angular deviations.

(2) The path gains, $\gamma_{k, j}(t), k=1,2, \ldots, K, j=1,2, \ldots, N_{k}$, $t=1,2, \ldots, T$, are temporally i.i.d. complex circularly symmetric zero-mean Gaussian random variables, whose covariance is

$\mathbb{E}\left\{\gamma_{k, j}(t) \gamma_{\tilde{k}, \tilde{j}}^{*}(\tilde{t})\right\}=\frac{\sigma_{\gamma_{k}}^{2}}{N_{k}} \delta(k-\tilde{k}) \delta(j-\tilde{j}) \delta(t-\tilde{t})$.

It can be seen from (5) that the path gain factors of different paths are uncorrelated, which corresponds to the rapidly time-varying channels. Consequently, the UTs are regarded as ID sources by the UCyA at the BS [10]. Moreover, in addition to the small-scale Rayleigh fading, the path gains also include the path loss and the shadow fading, which remain invariant during a single estimation period and influence the covariance $\sigma_{\gamma_{k}}^{2}$.

Note that as an example, in what follows we consider the case that each UT has only one pair of nominal DOAs, i.e., the nominal azimuth DOA and the nominal elevation DOA. However, when each UT has more than one pair of nominal DOAs, we can equivalently regard each UT as multiple ID sources obeying this assumption. Thus, the multiple pairs of nominal DOAs of each UT can also be estimated using the method proposed in this paper.

(3) The noise, $\mathbf{n}(t), t=1,2, \ldots, T$, are composed of temporally and spatially i.i.d. complex circularly symmetric zero-mean Gaussian variables, whose covariance matrix is given by

$\mathbb{E}\left\{\mathbf{n}(t) \mathbf{n}^{H}(\tilde{t})\right\}=\sigma_{\mathrm{n}}^{2} \mathbf{I}_{M} \delta(t-\tilde{t})$.

(4) The signals, $s_{k}(t), k=1,2, \ldots, K, t=1,2, \ldots, T$, are temporally i.i.d. zero-mean random variables with finite amplitudes and covariance

$\mathbb{E}\left\{s_{k}(t) s_{\tilde{k}}(\tilde{t})\right\}=\sigma_{s_{k}}^{2} \delta(k-\tilde{k}) \delta(t-\tilde{t})$.

Note that $\sigma_{s_{k}}^{2}$ also represents the transmitted power of the $k$ th UT. In addition, the signals are uncorrelated to the noise.

(5) The array is calibrated, which means the response of the array, i.e., the array steering vector $\mathbf{a}\left(\theta_{k, j}(t), \phi_{k, j}(t)\right)$ characterized in (2) for any given $\theta_{k, j}(t)$ and $\phi_{k, j}(t)$ is known a priori.

(6) The distance between any two adjacent antenna elements in the same UCA or in the same antenna column is less than or equal to $\lambda / 2$, which means $r \leq \lambda /(4 \sin (\pi / N))$ and $d \leq \lambda / 2$. This assumption assures that the received signal phases as well as the nominal DOAs, cf. (2), can be estimated without ambiguity.

\section{Beamspace transform}

The large number of BS antennas in massive MIMO systems entails high-dimensional received signal vectors. Thus, the computational complexity imposed is excessive. In this section, we first describe the phase mode excitation principle [40], which shows that the dimension of the array steering vector can be reduced when a phase-specific beamforming vector is employed. Then, we introduce the beamspace transform, which uses the phase mode excitation principle to reduce the dimensions of the received signal vectors. It is worth noting that the proposed approach can also be applied to other scenarios by exploiting the rotational invariance property of the antenna array's structure, such as uniform linear arrays (ULAs) and URAs.

\subsection{Phase mode excitation principle}

When the array is excited by a beamforming weight vector with a phase, the output of the array is related to this phase, and phase mode excitation principle is the relation between the output and the phase. We first consider the Ith UCA. According to (2), the array steering vector of the $l$ th UCA $\mathbf{a}_{l}\left(\theta_{k, j}(t), \phi_{k, j}(t)\right) \in \mathbb{C}^{N \times 1}$ is given by

$\left[\mathbf{a}_{l}\left(\theta_{k, j}(t), \phi_{k, j}(t)\right)\right]_{n}=\left[\mathbf{a}\left(\theta_{k, j}(t), \phi_{k, j}(t)\right)\right]_{N(l-1)+n}$,

where $n=1,2, \ldots, N$. It can be seen that the array steering vector of the lth UCA, $\mathbf{a}_{l}\left(\theta_{k, j}(t), \phi_{k, j}(t)\right)$, is constituted by $N$ consecutive elements of the array steering vector of the UCyA, $\mathbf{a}\left(\theta_{k, j}(t), \phi_{k, j}(t)\right)$. Here, the $N$ elements begin from $\left[\mathbf{a}\left(\theta_{k, j}(t), \phi_{k, j}(t)\right)\right]_{N(l-1)+1}$. Consider a beamforming weight vector

$\mathbf{w}_{p}=\frac{1}{N}\left[\exp \left(i p \vartheta_{1}\right), \exp \left(i p \vartheta_{2}\right), \ldots, \exp \left(i p \vartheta_{N}\right)\right]^{H} \in \mathbb{C}^{N \times 1}$,

where $p=-P,-P+1, \ldots, P$ is named the phase mode. The phase mode $p$ determines the phases $p \vartheta_{n}, n=1,2, \ldots, N$, in 
the vector $\mathbf{w}_{p}$. The value of the highest phase mode $P$ will be given later. Obviously, there are $P^{\prime}=2 P+1$ realizations of the beamforming weight vector $\mathbf{w}_{p}$. When the lth UCA is excited with the beamforming weight vector $\mathbf{w}_{p}$, the output of the array is named as the array pattern, and is expressed as

$f_{p, l}\left(\theta_{k, j}(t), \phi_{k, j}(t)\right)=\mathbf{w}_{p}^{H} \mathbf{a}_{l}\left(\theta_{k, j}(t), \phi_{k, j}(t)\right)$.

In this paper, $\mathbf{a}_{l}\left(\theta_{k, j}(t), \phi_{k j}(t)\right)$ and $\mathbf{x}_{l}(t)$ are in the element space, while $\mathbf{w}_{p}^{H} \mathbf{a}_{l}\left(\theta_{k, j}(t), \phi_{k, j}(t)\right)$ and $\mathbf{w}_{p}^{H} \mathbf{x}_{l}(t)$ are in the beamspace. Therefore, the beamforming weight vector $\mathbf{w}_{p}$ essentially performs the transform from the element space to the beamspace. For any phase mode $p$ that satisfies $|p|<N$, the array pattern can be further written as $[40,43]$

$$
\begin{aligned}
f_{p, l}\left(\theta_{k, j}(t), \phi_{k, j}(t)\right)= & {\left[i^{p} J_{p}\left(\zeta_{k, j}(t)\right) \exp \left(i p \theta_{k, j}(t)\right)+\varepsilon_{p}\left(\zeta_{k, j}(t), \theta_{k, j}(t)\right)\right] } \\
& \times \exp \left(-i \mu d(l-1) \cos \left(\phi_{k, j}(t)\right)\right),
\end{aligned}
$$

where $\zeta_{k, j}(t)=\mu r \sin \left(\phi_{k, j}(t)\right), J_{p}(\cdot)$ is the Bessel function of the first kind of order $p, i^{p} J_{p}\left(\zeta_{k, j}(t)\right) \exp \left(i p \theta_{k, j}(t)\right)$ is the principle term, and

$$
\begin{aligned}
\varepsilon_{p}\left(\zeta_{k, j}(t), \theta_{k, j}(t)\right)= & \sum_{q=1}^{\infty}\left[i^{g} J_{g}\left(\zeta_{k, j}(t)\right) \exp \left(-i g \theta_{k, j}(t)\right)\right. \\
& \left.+i^{h} J_{h}\left(\zeta_{k, j}(t)\right) \exp \left(i h \theta_{k, j}(t)\right)\right]
\end{aligned}
$$

is the residual term, in which $g=N q-p$ and $h=N q+p$.

In massive MIMO systems, the number of antennas of each UCA, $N$, is very large. When $P>\mu r$ and $N>2 P$ are satisfied, ${ }^{2}$ the residual term $\varepsilon_{p}\left(\zeta_{k, j}(t), \theta_{k, j}(t)\right)$ is much smaller than the principal term $i^{p} J_{p}\left(\zeta_{k, j}(t)\right) \exp \left(i p \theta_{k, j}(t)\right)$ for any azimuth DOA $\theta_{k, j}(t)$ and elevation DOA $\phi_{k, j}(t)$ [40]. Obviously, when $N>2 P$ and $P>\mu r$, the array pattern $f_{p, l}\left(\theta_{k, j}(t), \phi_{k, j}(t)\right)$ and its partial derivatives can be approximated by neglecting the residual term as

$$
\begin{aligned}
& f_{p, l}\left(\theta_{k, j}(t), \phi_{k, j}(t)\right) \approx i^{p} J_{p}\left(\zeta_{k, j}(t)\right) \exp \left(i p \theta_{k, j}(t)\right) \\
& \quad \times \exp \left(-i \mu d(l-1) \cos \left(\phi_{k, j}(t)\right)\right), \\
& \frac{\partial f_{p, l}\left(\theta_{k, j}(t), \phi_{k, j}(t)\right)}{\partial \theta_{k, j}(t)} \approx i^{p+1} p J_{p}\left(\zeta_{k, j}(t)\right) \exp \left(i p \theta_{k, j}(t)\right) \\
& \quad \times \exp \left(-i \mu d(l-1) \cos \left(\phi_{k, j}(t)\right)\right),
\end{aligned}
$$

and

$$
\begin{aligned}
& \frac{\partial f_{p, l}\left(\theta_{k, j}(t), \phi_{k, j}(t)\right)}{\partial \phi_{k, j}(t)} \approx i^{p}\left(J_{p-1}\left(\zeta_{k, j}(t)\right)-J_{p+1}\left(\zeta_{k, j}(t)\right)\right) \\
& \quad \times \exp \left(i p \theta_{k, j}(t)\right) \exp \left(-i \mu d(l-1) \cos \left(\phi_{k, j}(t)\right)\right) \cos \left(\phi_{k, j}(t)\right) \\
& \quad \times \frac{1}{2} \mu r+f_{p, l}\left(\theta_{k, j}(t), \phi_{k, j}(t)\right) i \mu d(l-1) \sin \left(\phi_{k, j}(t)\right),
\end{aligned}
$$

respectively. It is known that there are $P^{\prime}$ different realizations of $f_{p, l}\left(\theta_{k, j}(t), \phi_{k, j}(t)\right)$, while there are $N$ elements in the array steering vector $\mathbf{a}_{l}\left(\theta_{k, j}(t), \phi_{k, j}(t)\right)$. This means that

\footnotetext{
${ }^{2}$ Since $\mu=2 \pi / \lambda$, we have $\mu r=2 \pi r / \lambda$. In wireless communication systems, the radius of the UCA, $r$, changes in accordance with the wavelength, $\lambda$. For example, in the macrocell scenario, the wavelength and the radius are long; while in the future small cell scenario, the wavelength and the radius are short. Thus, the number of UCA antennas, $N$, can satisfy $N>2 P$ and $P>\mu r$ in massive MIMO systems.
}

the dimension $N$ of the array steering vector of each UCA in the element space $N$ is changed to the dimension $P^{\prime}$ in the beamspace. Therefore, the beamspace transform is capable of remarkably reducing the dimension of the array steering vector in the context of massive MIMO systems.

Relying on the above-mentioned properties of the phase mode excitation, in what follows we will proceed to transform the received signal vectors from the element space into the beamspace, and will develop a beamspace approach for estimating 2-D angular parameters in massive MIMO systems.

\subsection{Beamspace transform for the UCyA}

The phase mode excitation principle may be employed to transform the received signal vectors from the element space into the beamspace, and the corresponding transformation is known as the beamspace transform; thus, the dimensions of the received signal vectors can also be significantly reduced in massive MIMO systems. According to (1), the received signal vector of the lth UCA is given by

$\mathbf{x}_{l}(t)=\sum_{k=1}^{K} s_{k}(t) \sum_{j=1}^{N_{k}} \gamma_{k j}(t) \mathbf{a}_{l}\left(\theta_{k, j}(t), \phi_{k, j}(t)\right)+\mathbf{n}_{l}(t) \in \mathbb{C}^{N \times 1}$,

where $\mathbf{a}_{l}\left(\theta_{k, j}(t)\right)$ is defined in (8), and the received noise vector $\mathbf{n}_{l}(t) \in \mathbb{C}^{N \times 1}$ of the lth UCA is characterized by $\left[\mathbf{n}_{l}(t)\right]_{n}=[\mathbf{n}(t)]_{N(l-1)+n}$, where $n=1,2, \ldots, N$.

According to (10), we can transform the received signal vector $\mathbf{x}_{l}(t)$ from the element space into the beamspace, namely we have

$$
\begin{aligned}
\tilde{\mathbf{x}}_{l}(t)= & \mathbf{F}_{\mathrm{e}}^{H} \mathbf{x}_{l}(t)=\sum_{k=1}^{K} s_{k}(t) \sum_{j=1}^{N_{k}} \gamma_{k, j}(t) \mathbf{b}_{l}\left(\theta_{k, j}(t), \phi_{k, j}(t)\right) \\
& +\tilde{\mathbf{n}}_{l}(t) \in \mathbb{C}^{P^{\prime} \times 1},
\end{aligned}
$$

where

$\mathbf{F}_{\mathrm{e}}=\sqrt{N}\left[i^{-P} \mathbf{w}_{-P}, i^{-P+1} \mathbf{w}_{-P+1}, \ldots, i^{P} \mathbf{w}_{P}\right] \in \mathbb{C}^{N \times P^{\prime}}$

is the beamforming matrix as defined in [40]; $\mathbf{b}_{l}\left(\theta_{k, j}(t)\right.$, $\left.\phi_{k, j}(t)\right) \in \mathbb{C}^{P^{\prime} \times 1}$ is the beamspace array steering vector of the lth UCA, and it is defined as

$\left[\mathbf{b}_{l}\left(\theta_{k, j}(t), \phi_{k, j}(t)\right)\right]_{p+P+1}=\sqrt{N} i^{-p} f_{p, l}\left(\theta_{k, j}(t), \phi_{k, j}(t)\right)$,

$p=-P,-P+1, \ldots, P ; \quad$ and $\quad \tilde{\mathbf{n}}_{l}(t)=\mathbf{F}_{\mathrm{e}}^{H} \mathbf{n}_{l}(t) \in \mathbb{C}^{P^{\prime} \times 1}$ is the beamspace received noise vector of the lth UCA. Comparing (8) and (18), we can see that the array steering vector of the $l$ th UCA, $\mathbf{a}_{l}\left(\theta_{k, j}(t), \phi_{k, j}(t)\right)$, is completely different from the beamspace array steering vector of the lth UCA, $\mathbf{b}_{l}\left(\theta_{k, j}(t), \phi_{k, j}(t)\right)$. According to the definitions of $\mathbf{w}_{p}$ in (9) and $\mathbf{F}_{\mathrm{e}}$ in (17), we have $\mathbf{F}_{\mathrm{e}}^{H} \mathbf{F}_{\mathrm{e}}=\mathbf{I}_{P^{\prime}}$. Hence, the noise vectors $\tilde{\mathbf{n}}_{l}(t), l=1,2, \ldots, L$, in the beamspace are also spatially and temporally white zero-mean Gaussian noise vectors with covariance matrix $\sigma_{\mathrm{n}}^{2} \mathbf{I}_{P^{\prime}}$.

Then, the beamspace received signal vectors of all the $L$ UCAs are stacked into a vector, which is defined as the beamspace received signal vector of the UCyA, and is given by

$\tilde{\mathbf{x}}(t)=\left[\tilde{\mathbf{x}}_{1}^{T}(t), \tilde{\mathbf{x}}_{2}^{T}(t), \ldots, \tilde{\mathbf{x}}_{L}^{T}(t)\right]^{T}$ 


$$
=\sum_{k=1}^{K} s_{k}(t) \sum_{j=1}^{N_{k}} \gamma_{k, j}(t) \mathbf{b}\left(\theta_{k, j}(t), \phi_{k, j}(t)\right)+\tilde{\mathbf{n}}(t)
$$

$\tilde{\mathbf{x}}(t) \in \mathbb{C}^{M^{\prime} \times 1}$,

where $M^{\prime}=P^{\prime} L$ is the dimension of the beamspace received signal vector of the UCyA, and

$$
\begin{aligned}
& \mathbf{b}\left(\theta_{k, j}(t), \phi_{k, j}(t)\right)=\left[\mathbf{b}_{1}^{T}\left(\theta_{k, j}(t), \phi_{k, j}(t)\right), \mathbf{b}_{2}^{T}\left(\theta_{k, j}(t), \phi_{k, j}(t)\right), \ldots,\right. \\
&\left.\mathbf{b}_{L}^{T}\left(\theta_{k, j}(t), \phi_{k, j}(t)\right)\right]^{T} \in \mathbb{C}^{M^{\prime} \times 1}
\end{aligned}
$$

is the beamspace array steering vector of the UCyA. Since the array steering vector of the lth UCA is completely different from the beamspace array steering vector of the $l$ th UCA, the array steering vector of the UCyA given by (2) is also completely different from the beamspace array steering vector of the UCyA defined here. It should be noticed that the total dimension of the received signal vector of the UCyA in the beamspace is $M^{\prime}$, and is significantly smaller than the total dimension $M=N L$ of the received signal vector of the UCyA in the element space. Hence, for large $N$, the total dimensions of the received signal vectors of the UCyA are greatly reduced after using the beamspace transform, which facilitates employing localization algorithms in massive MIMO systems. In the next section, the beamspace received signal vectors given by (20) will be invoked to estimate 2-D angular parameters.

\section{The generalized beamspace approach}

The existing method in [34] estimates angular parameters in the element space. The large dimensions of received signal vectors in massive MIMO systems make this method excessively complicated. Hence, in this section, a generalized beamspace ESPRIT approach and a generalized beamspace MUSIC approach are proposed for estimating nominal elevation and azimuth DOAs, respectively. As a beneficial result of the beamspace transform, the proposed methods enjoy significantly lower computational complexity than the existing element space method in [34].

\subsection{Estimation of nominal elevation DOAs: generalized beamspace ESPRIT}

The classical ESPRIT approach [36-38] has lower computational complexity than the other existing approaches conceived for 2-D localization of ID sources, such as the ML approach, the approximated ML approach, and the LSbased covariance matching approach. However, the search dimensions of all these methods remain too high for practical implementation, especially in the context of massive MIMO systems. Notably, by exploiting beamspace transform, the computational complexity of 2-D localization of ID sources can be further reduced compared to that of using the conventional element-space based ESPRIT approach. Relying on the beamspace received signal vectors, the nominal elevation DOAs can be estimated by generalizing the ESPRIT approach to 2-D ID sources. Thus, the computational complexity of the estimation of nominal elevation DOAs is very low.

Since we only have the knowledge of the received signal vector and aim to estimate the 2-D nominal DOAs, the relation between the received signal vector and the 2D nominal DOAs will be derived. For small angular deviations $\tilde{\theta}_{k, j}(t)$ and $\tilde{\phi}_{k, j}(t)$, the beamspace array steering vector $\mathbf{b}\left(\theta_{k, j}(t), \phi_{k, j}(t)\right)$ of the UCyA may be expressed by the Taylor series expansion as

$$
\begin{aligned}
\mathbf{b}\left(\theta_{k, j}(t), \phi_{k, j}(t)\right)= & \mathbf{b}\left(\bar{\theta}_{k}, \bar{\phi}_{k}\right)+\frac{\partial \mathbf{b}\left(\bar{\theta}_{k}, \bar{\phi}_{k}\right)}{\partial \bar{\theta}_{k}} \tilde{\theta}_{k, j}(t) \\
& +\frac{\partial \mathbf{b}\left(\bar{\theta}_{k}, \bar{\phi}_{k}\right)}{\partial \bar{\phi}_{k}} \tilde{\phi}_{k, j}(t)+\epsilon_{k, j}(t),
\end{aligned}
$$

where $\epsilon_{k, j}(t)$ is the least significant term. Here we omit this term $\epsilon_{k, j}(t)$, and the error introduced is negligible for small angular deviations. ${ }^{3}$ According to (20), the beamspace received signal vector of the UCyA can be expressed as

$$
\begin{aligned}
\tilde{\mathbf{x}}(t)= & \sum_{k=1}^{K}\left(\mathbf{b}\left(\bar{\theta}_{k}, \bar{\phi}_{k}\right) c_{k, 1}+\frac{\partial \mathbf{b}\left(\bar{\theta}_{k}, \bar{\phi}_{k}\right)}{\partial \bar{\theta}_{k}} c_{k, 2}+\frac{\partial \mathbf{b}\left(\bar{\theta}_{k}, \bar{\phi}_{k}\right)}{\partial \bar{\phi}_{k}} c_{k, 3}\right) \\
& +\tilde{\mathbf{n}}(t),
\end{aligned}
$$

where we have $c_{k, 1}(t)=s_{k}(t) \sum_{j=1}^{N_{k}} \gamma_{k, j}(t), \quad c_{k, 2}(t)=s_{k}(t)$ $\sum_{j=1}^{N_{k}} \gamma_{k, j}(t) \tilde{\theta}_{k, j}(t)$, and $c_{k, 3}(t)=s_{k}(t) \sum_{j=1}^{N_{k}} \gamma_{k, j}(t) \tilde{\phi}_{k, j}(t)$. For the sake of clarity, the beamspace received signal vector of the UCyA is reformulated in a compact matrix form as

$\tilde{\mathbf{x}}(t)=\mathbf{A c}(t)+\tilde{\mathbf{n}}(t)$,

where

$$
\begin{aligned}
& \mathbf{A}=\left[\mathbf{b}\left(\bar{\theta}_{1}, \bar{\phi}_{1}\right), \mathbf{b}\left(\bar{\theta}_{2}, \bar{\phi}_{2}\right), \ldots, \mathbf{b}\left(\bar{\theta}_{K}, \bar{\phi}_{K}\right), \frac{\partial \mathbf{b}\left(\bar{\theta}_{1}, \bar{\phi}_{1}\right)}{\partial \bar{\theta}_{1}},\right. \\
& \frac{\partial \mathbf{b}\left(\bar{\theta}_{2}, \bar{\phi}_{2}\right)}{\partial \bar{\theta}_{2}}, \ldots, \frac{\partial \mathbf{b}\left(\bar{\theta}_{K}, \bar{\phi}_{K}\right)}{\partial \bar{\theta}_{K}}, \frac{\partial \mathbf{b}\left(\bar{\theta}_{1}, \bar{\phi}_{1}\right)}{\partial \bar{\phi}_{1}}, \\
&\left.\frac{\partial \mathbf{b}\left(\bar{\theta}_{2}, \bar{\phi}_{2}\right)}{\partial \bar{\phi}_{2}}, \ldots, \frac{\partial \mathbf{b}\left(\bar{\theta}_{K}, \bar{\phi}_{K}\right)}{\partial \bar{\phi}_{K}}\right] \in \mathbb{C}^{M^{\prime} \times 3 K}
\end{aligned}
$$

is named as the beamspace array response matrix, and the elements of

$$
\begin{aligned}
\mathbf{c}(t)= & {\left[c_{1,1}(t), c_{2,1}(t), \ldots, c_{K, 1}(t), c_{1,2}(t), c_{2,2}(t), \ldots,\right.} \\
& \left.c_{K, 2}(t), c_{1,3}(t), c_{2,3}(t), \ldots, c_{K, 3}(t)\right]^{T} \in \mathbb{C}^{3 K \times 1}
\end{aligned}
$$

are functions of the transmitted signal, the path gains, and the angular deviations.

From the above analysis, it can be seen that the received signal vector is related to both the 2-D nominal DOAs and some random variables. Thus, the relation given by (22) cannot be directly employed to estimate the nominal DOAs. Inspired by the fact that the randomness can be removed with expectation, we derive the covariance matrix of the beamspace received signal vector of the UCyA, which is given by

$\mathbf{R}_{\tilde{\mathbf{x}}}=\mathbb{E}\left\{\tilde{\mathbf{x}}(t) \tilde{\mathbf{X}}^{H}(t)\right\}=\mathbf{A} \mathbf{\Lambda}_{\mathrm{c}} \mathbf{A}^{H}+\sigma_{\mathrm{n}}^{2} \mathbf{I}_{M^{\prime}} \in \mathbb{C}^{M^{\prime} \times M^{\prime}}$,

\footnotetext{
${ }^{3}$ The angular spreads of the sources as seen from the base station are small in most circumstances [50,51]. Therefore, only the small angular spreads are considered in this paper. The scenario where sources have large angular spread will be addressed in our future work.
} 
where $\boldsymbol{\Lambda}_{c}=\mathbb{E}\left\{\mathbf{c}(t) \mathbf{c}^{H}(t)\right\} \in \mathbb{C}^{3 K \times 3 K}$. Based on the properties of $\tilde{\theta}_{k, j}(t), \tilde{\phi}_{k, j}(t), \gamma_{k, j}(t)$, and $s_{k}(t)$ given in (3)-(5) and (7), we can see that $\boldsymbol{\Lambda}_{c}$ is a diagonal matrix with $\left[\boldsymbol{\Lambda}_{c}\right]_{k, k}=\sigma_{s_{k}}^{2} \sigma_{\gamma_{k}}^{2}$, $\left[\boldsymbol{\Lambda}_{\mathrm{c}}\right]_{K+k, K+k}=\left[\boldsymbol{\Lambda}_{\mathrm{c}}\right]_{k, k} \sigma_{\theta_{k}}^{2}$, and $\left[\boldsymbol{\Lambda}_{\mathrm{c}}\right]_{2 K+k, 2 K+k}=\left[\boldsymbol{\Lambda}_{\mathrm{c}}\right]_{k, k} \sigma_{\phi_{k}}^{2}, k=$ $1,2, \ldots, K$. According to (24), we obtain the eigenvaluedecomposition (EVD) of $\mathbf{R}_{\tilde{\mathbf{x}}}$ as follows:

$\mathbf{R}_{\tilde{\mathbf{x}}}=\mathbf{E}_{\mathrm{s}} \boldsymbol{\Sigma}_{\mathrm{s}} \mathbf{E}_{\mathrm{s}}^{H}+\sigma_{\mathrm{n}}^{2} \mathbf{E}_{\mathrm{n}} \mathbf{E}_{\mathrm{n}}^{H}$,

where $\mathbf{E}_{\mathrm{s}} \in \mathbb{C}^{M^{\prime} \times 3 K}$ corresponds to the signal subspace of the UCyA, $\mathbf{E}_{\mathrm{n}} \in \mathbb{C}^{M^{\prime} \times\left(M^{\prime}-3 K\right)}$ corresponds to the noise subspace of the UCyA, and $\boldsymbol{\Sigma}_{\mathrm{s}} \in \mathbb{R}^{3 K \times 3 K}$ is a diagonal matrix whose diagonal elements are the largest $3 K$ eigenvalues of $\mathbf{R}_{\tilde{\mathbf{x}}}$. Obviously, the linear relation

$\mathbf{E}_{\mathrm{S}}=\mathbf{A T}$

can be obtained, where $\mathbf{T} \in \mathbb{C}^{3 K \times 3 K}$ is a matrix of full rank. It can be seen that this linear relation is much simpler than that given by (22). However, T in (26) is not known. Hence, the relation of (26) cannot be immediately used to estimate the nominal DOAs.

In order to get rid of the impact of $\mathbf{T}$ on the estimation, we will derive the linear relation amongst the submatrices of $\mathbf{A}$, and the submatrices of $\mathbf{A}$ are related to the 2-D nominal DOAs. First, we derive the expressions of the elements of $\mathbf{A}$, then we derive the linear relation between submatrices of $\mathbf{A}$.

Proposition 1. When $P$, namely the value of the highest phase mode, satisfies $\mu r<P<N / 2$, the linear recurrence relation between the beamspace array response matrices of each pair of UCAs can be expressed as

$\mathbf{A}_{l^{\prime}+1}=\mathbf{A}_{l^{\prime}} \Phi$,

where

$\mathbf{A}_{l}=\mathbf{J}_{l} \mathbf{A} \in \mathbb{C}^{P^{\prime} \times P^{\prime}}$

is the beamspace array response matrix of the l'th UCA and it is a submatrix of $\mathbf{A}$, while

$\mathbf{J}_{l}=\left[\mathbf{0}_{P^{\prime} \times P^{\prime}(l-1)}, \mathbf{I}_{P^{\prime}}, \mathbf{0}_{P^{\prime} \times P^{\prime}(L-l)}\right] \in \mathbb{R}^{P^{\prime} \times M^{\prime}}$

is the corresponding selection matrix that selects $P^{\prime}$ rows from A to construct $\mathbf{A}_{l}$. (27) is obtained by exploiting the Taylor series expansion and the beamspace transform. In addition,

$\boldsymbol{\Phi}=\left[\begin{array}{lll}\boldsymbol{\Lambda}_{0} & \mathbf{0}_{K \times K} & \boldsymbol{\Lambda}_{1} \\ \mathbf{0}_{K \times K} & \boldsymbol{\Lambda}_{0} & \mathbf{0}_{K \times K} \\ \mathbf{0}_{K \times K} & \mathbf{0}_{K \times K} & \boldsymbol{\Lambda}_{0}\end{array}\right] \in \mathbb{C}^{3 K \times 3 K}$

is an upper triangular matrix, in which

$$
\begin{aligned}
\boldsymbol{\Lambda}_{0}= & \operatorname{diag}\left(\exp \left(-i \mu d \cos \left(\bar{\phi}_{1}\right)\right), \exp \left(-i \mu d \cos \left(\bar{\phi}_{2}\right)\right), \ldots,\right. \\
& \left.\exp \left(-i \mu d \cos \left(\bar{\phi}_{K}\right)\right)\right) \in \mathbb{C}^{K \times K}
\end{aligned}
$$

and

$\boldsymbol{\Lambda}_{1}=i \mu d \operatorname{diag}\left(\sin \left(\bar{\phi}_{1}\right), \sin \left(\bar{\phi}_{2}\right), \ldots, \sin \left(\bar{\phi}_{K}\right)\right) \boldsymbol{\Lambda}_{0} \in \mathbb{C}^{K \times K}$.

Proof. See Appendix A.

Remark 1. $\Lambda_{1}$ in (29) was approximated as a zero matrix in [34]. By contrast, in this paper, the linear relation in (27) is derived rigorously without omitting $\boldsymbol{\Lambda}_{1}$. Relying on (27), we can employ (26) to construct a similar linear relation amongst the submatrices of the signal subspace matrix $\mathbf{E}_{\mathrm{s}}$, and this relation may be obtained from the beamspace received signal vectors of the UCyA. As a result, we can estimate the nominal elevation DOAs by invoking the linear relation amongst the submatrices of $\mathbf{E}_{\mathrm{s}}$.

From (26) and (28), the submatrix of the signal subspace matrix $\mathbf{E}_{\mathrm{s}}$ of the UCyA is given by

$\mathbf{E}_{l}=\mathbf{J}_{l} \mathbf{E}_{\mathrm{s}}$

$\mathbf{E}_{l}=\mathbf{A}_{l} \mathbf{T} \in \mathbb{C}^{P^{\prime} \times 3 K}$

Substituting (27) into (32) yields

$\mathbf{E}_{l^{\prime}+1}=\mathbf{A}_{l^{\prime}} \mathbf{\Phi} \mathbf{T}=\mathbf{E}_{l^{\prime}} \mathbf{T}^{-1} \mathbf{\Phi T}$,

where the second equation is based on $\mathbf{A}_{l^{\prime}}=\mathbf{E}_{l^{\prime}} \mathbf{T}^{-1}$.

From the definition of $\mathbf{E}_{l}$ in (32), it is known that the rank of $\mathbf{E}_{l}$ is the smaller value between $P^{\prime}$ and $3 K$. As long as $P^{\prime} \geq 3 K, \quad \boldsymbol{\Psi}=\mathbf{T}^{-1} \mathbf{\Phi T} \in \mathbb{C}^{3 K \times 3 K}$ can be estimated from $\mathbf{E}_{l^{\prime}+1}$ and $\mathbf{E}_{l^{\prime}}$ by employing the well-known total leastsquares (TLS) criterion [38], and the estimate is denoted as $\hat{\mathbf{\Psi}}_{l^{\prime}}, l^{\prime}=1,2, \ldots, L-1$. Since the eigenvalues of an upper triangular matrix are also the diagonal elements of this matrix, the sorted eigenvalues of $\hat{\mathbf{\Psi}}_{l^{\prime}}$, which are denoted as $\lambda_{l^{\prime}, k^{\prime}}, k^{\prime}=1,2, \ldots, 3 K$, are taken as the estimates of the diagonal elements of $\boldsymbol{\Phi}$. Because there are $(L-1)$ different estimates of the matrix $\boldsymbol{\Psi}$, i.e., $\hat{\mathbf{\Psi}}_{l^{\prime}}, l^{\prime}=1,2, \ldots, L-1$, there are $(L-1)$ different estimates for each diagonal element of $\boldsymbol{\Phi}$, thus there are $3(L-1)$ different estimates for each diagonal element of $\boldsymbol{\Lambda}_{0}$. According to (30), the nominal elevation DOA of the $k$ th UT is estimated as

$\hat{\bar{\phi}}_{k}=\frac{1}{3(L-1)} \sum_{l^{\prime}=1}^{L-1} \sum_{k^{\prime}=3 k-2}^{3 k} \arccos \left(\frac{i \ln \lambda_{l^{\prime}, k^{\prime}}}{\mu d}\right)$.

It can be seen that the nominal elevation DOAs are estimated by using the linear relation in (27). Hence, the approximations in [34] for omitting $\boldsymbol{\Lambda}_{1}$ in (29) are not necessary.

It can be seen that the beamspace transform is used to derive linear relations, which are critical to the employment of the ESPRIT approach. Note that no direct derivation of these linear relations was available in the open literature. After the estimates of the nominal elevation DOAs have been obtained, they can be used to estimate nominal azimuth DOAs. In Section 4.2, the proposed search algorithm will be detailed.

\subsection{Estimation of nominal azimuth DOAs: generalized beamspace MUSIC}

From the previous derivation, we can see that the linear relation of (27) is crucial to estimating the nominal elevation DOAs with the generalized beamspace ESPRIT. However, it can be easily found that there is no similar relation for the nominal azimuth DOAs. Thus, we cannot proceed to estimate the nominal azimuth DOAs with the generalized beamspace ESPRIT. Fortunately, the beamspace MUSIC method [40] has been shown to achieve good performance for point sources at the expense of slightly higher computational complexity than the beamspace ESPRIT. Hence, the 
beamspace MUSIC for point sources can be generalized for estimating nominal azimuth DOAs of ID sources.

In order to reduce computational complexity, only the beamspace received signal vector of the first UCA, i.e., $\tilde{\mathbf{x}}_{1}(t)$ in (19), is used for estimation. From (19) and (24), it can be seen that the covariance matrix of $\tilde{\mathbf{x}}_{1}(t)$ corresponds to the $P^{\prime} \times P^{\prime}$ submatrix in the upper left corner of $\mathbf{R}_{\tilde{\mathbf{x}}}$, and is denoted as $\mathbf{R}_{\tilde{\mathbf{x}}_{1}} \in \mathbb{C}^{P^{\prime} \times P^{\prime}}$ here. Then, we have

$\mathbf{R}_{\tilde{\mathbf{x}}_{1}}=\mathbf{A}_{1} \boldsymbol{\Lambda}_{\mathrm{c}} \mathbf{A}_{1}^{H}+\sigma_{\mathrm{n}}^{2} \mathbf{I}_{P^{\prime}}$

where $\mathbf{A}_{1}$ is defined in (28). Similar to (25), the EVD of $\mathbf{R}_{\tilde{\mathbf{x}}_{1}}$ is expressed as

$\mathbf{R}_{\tilde{\mathbf{x}}_{1}}=\mathbf{E}_{\mathrm{s}_{1}} \boldsymbol{\Sigma}_{\mathrm{s}_{1}} \mathbf{E}_{\mathrm{s}_{1}}^{H}+\sigma_{\mathrm{n}}^{2} \mathbf{E}_{\mathrm{n}_{1}} \mathbf{E}_{\mathrm{n}_{1}}^{H}$,

where $\mathbf{E}_{\mathrm{s}_{1}} \in \mathbb{C}^{P^{\prime} \times 3 K}$ and $\mathbf{E}_{\mathrm{n}_{1}} \in \mathbb{C}^{P^{\prime} \times\left(P^{\prime}-3 K\right)}$ correspond to the signal subspace and noise subspace of the first UCA, respectively, and $\boldsymbol{\Sigma}_{\mathrm{s}_{1}} \in \mathbb{R}^{3 K \times 3 K}$ is a diagonal matrix whose diagonal elements are the largest $3 K$ eigenvalues of $\mathbf{R}_{\tilde{\mathbf{x}}_{1}}$. Obviously, we have

$\mathbf{E}_{\mathrm{S}_{1}}=\mathbf{A}_{1} \mathbf{T}_{1}$,

where $\mathbf{T}_{1} \in \mathbb{C}^{3 K \times 3 K}$ is a matrix of full rank. Then, the orthogonality between the columns of $\mathbf{E}_{\mathrm{s}_{1}}$ and that of $\mathbf{E}_{\mathrm{n}_{1}}$, or equivalently the orthogonality between the columns of $\mathbf{A}_{1}$ and that of $\mathbf{E}_{\mathrm{n}_{1}}$, can be exploited to derive the generalized beamspace MUSIC spectrum

$Q\left(\bar{\theta}, \hat{\bar{\phi}}_{k}\right)=\frac{1}{\left\|\mathbf{E}_{\mathrm{n}_{1}}^{H} \mathbf{q}\left(\bar{\theta}, \hat{\bar{\phi}}_{k}\right)\right\|_{\mathrm{F}}^{2}}$,

where

$\mathbf{q}\left(\bar{\theta}, \hat{\bar{\phi}}_{k}\right)=\left[\mathbf{b}_{1}\left(\bar{\theta}, \hat{\bar{\phi}}_{k}\right), \partial \mathbf{b}_{1}\left(\bar{\theta}, \hat{\bar{\phi}}_{k}\right) / \partial \bar{\theta}, \partial \mathbf{b}_{1}\left(\bar{\theta}, \hat{\bar{\phi}}_{k}\right) / \partial \hat{\bar{\phi}}_{k}\right] \in \mathbb{C}^{P^{\prime} \times 3}$

is calculated by using (46), (48), and (50), and $\bar{\theta}$ is the azimuth DOA that will be estimated by search. With this spectrum, the nominal azimuth DOA of the $k$ th UT is estimated by using the 1-D search as

$\hat{\bar{\theta}}_{k}=\arg \max _{\bar{\theta}} Q\left(\bar{\theta}, \hat{\bar{\phi}}_{k}\right)$

It can be seen from (38) that only 1-D search is required for estimating the nominal azimuth DOA, and the computational complexity imposed is lower than that of the generalized MUSIC (GMUSIC) approach in [34] due to the employment of the beamspace transform. ${ }^{4}$

\subsection{Estimation of angular spreads}

2-D angular spreads of the $K$ UTs can be estimated after the nominal azimuth and elevation DOAs have been obtained. According to (35), the estimator of $\boldsymbol{\Lambda}_{c}$ is formulated as

$\hat{\mathbf{\Lambda}}_{\mathrm{c}}=\hat{\mathbf{A}}_{1}^{\dagger}\left(\mathbf{R}_{\tilde{\mathbf{x}}_{1}}-\hat{\sigma}_{\mathrm{n}}^{2} \mathbf{I}_{P^{\prime}}\right)\left(\hat{\mathbf{A}}_{1}^{H}\right)^{\dagger} \in \mathbb{C}^{3 K \times 3 K}$,

where $\hat{\mathbf{A}}_{1} \in \mathbb{C}^{P^{\prime} \times 3 K}$ is obtained by replacing $\bar{\theta}_{k}$ and $\bar{\phi}_{k}$ in $\mathbf{A}_{1}$, cf. (23) and (28), with $\hat{\bar{\theta}}_{k}$ and $\hat{\bar{\phi}}_{k}$, respectively. From the definition of $\mathbf{E}_{\mathrm{n}_{1}}$ below (36), it is known that the noise

${ }^{4}$ The dimensions of $\overline{\mathbf{a}}_{1}\left(\bar{\theta}, \hat{\bar{\phi}}_{k}\right)$ here are $P^{\prime}$ and 3, while the dimensions of $\overline{\mathbf{a}}_{1}\left(\bar{\theta}, \hat{\bar{\phi}}_{k}\right)$ in [34] are $M$ and 3 , and $M \gg P^{\prime}$. subspace exists only when $P^{\prime}>3 K$ is satisfied. As long as $P^{\prime}>3 K$, the estimate of the variance $\hat{\sigma}_{\mathrm{n}}^{2}$ of the noise is obtained by averaging the smallest $P^{\prime}-3 K$ eigenvalues of $\mathbf{R}_{\tilde{\mathbf{x}}_{1}}$. According to the definition of the elements of $\boldsymbol{\Lambda}_{\mathrm{c}}$ given below (24), the angular spreads can be estimated as

$\hat{\sigma}_{\theta_{k}}=\sqrt{\frac{\left[\hat{\boldsymbol{\Lambda}}_{\mathrm{c}}\right]_{K+k, K+k}}{\left[\hat{\boldsymbol{\Lambda}}_{\mathrm{c}}\right]_{k, k}}}$

and

$\hat{\sigma}_{\phi_{k}}=\sqrt{\frac{\left[\hat{\boldsymbol{\Lambda}}_{\mathrm{c}}\right]_{2 K+k, 2 K+k}}{\left[\hat{\boldsymbol{\Lambda}}_{\mathrm{c}}\right]_{k, k}}}$,

respectively.

In practice, based on the ergodicity, the covariance matrix $\mathbf{R}_{\tilde{\mathbf{x}}}$ in (24) can be estimated as

$\hat{\mathbf{R}}_{\tilde{\mathbf{x}}}=\frac{1}{T} \sum_{t=1}^{T} \tilde{\mathbf{x}}(t) \tilde{\mathbf{x}}^{H}(t) \in \mathbb{C}^{M^{\prime} \times M^{\prime}}$.

Similarly, the $P^{\prime} \times P^{\prime}$ submatrix in the upper left corner of $\hat{\mathbf{R}}_{\tilde{\mathbf{x}}}$, which is denoted as $\hat{\mathbf{R}}_{\tilde{\mathbf{x}}_{1}}$, is taken as the estimate of $\mathbf{R}_{\tilde{\mathbf{x}}_{1}}$ in (36).

For the sake of clarity, the proposed generalized beamspace estimation approach, including the generalized ESPRIT, the generalized MUSIC, and the estimation of angular spreads, is summarized as follows.

Algorithm 1. Estimating the nominal DOAs and the angular spreads.

Step 1) Transform the received signal vectors from element space to beamspace and obtain $\tilde{\mathbf{x}}(t)$ by (16) and (19).

Step 2) Calculate the sample covariance matrix, $\hat{\mathbf{R}}_{\mathbf{x}}$, according to (42).

Step 3) Perform EVD on $\hat{\mathbf{R}}_{\mathbf{x}}$ according to (25) and divide the signal subspace matrix $\mathbf{E}_{\mathrm{s}}$ of the UCyA into $\mathbf{E}_{l}, l=1,2, \ldots, L$, according to (31), then employ the TLS criterion to estimate $\hat{\mathbf{\Psi}}_{l^{\prime}}, l^{\prime}=1,2, \ldots, L-1$

Step 4) Perform EVD on $\hat{\mathbf{\Psi}}_{l^{\prime}}, l^{\prime}=1,2, \ldots, L-1$, and obtain $\lambda_{I^{\prime}, K^{\prime}}, k^{\prime}=1,2, \ldots, 3 K$, then estimate $\hat{\bar{\phi}}_{k}, k=1,2, \ldots, K$ using (34).

Step 5) Perform EVD on $\mathbf{R}_{\tilde{\mathbf{x}}_{1}}$ according to (36), and estimate $\hat{\bar{\theta}}_{k}, k=1,2, \ldots, K$, with $1-D$ search using (38).

Step 6) Estimate $\hat{\boldsymbol{\Lambda}}_{\mathrm{c}}$ by (39), then estimate $\hat{\sigma}_{\theta_{k}}$ and $\hat{\sigma}_{\phi_{k}}$ using (40) and (41), respectively.

Remark 2. As opposed to the traditional element space approaches, such as the LS-based [17,26,27] and the MLbased $[17,18]$ approaches, as well as the existing GMUSIC approach [34], the proposed method estimate angular parameters in the beamspace rather than in the element space. Since the received signal vectors in the beamspace are of much lower dimensions, the proposed method exhibits significantly lower computational complexity than the element space approaches.

According to the statement $P^{\prime}>\mu r$ above (12), and the statements $P^{\prime} \geq 3 K$ and $P^{\prime}>3 K$ below (33) and (39), the dimension $P^{\prime}$ of the beamspace signal vectors is only required to be larger than both $2 \mu r+1$ and $3 K$, thus $P^{\prime}$ is much smaller than the number of antennas of any large UCA. The computational complexity of the proposed approach and of the existing GMUSIC method will be analyzed in more detail in the next section. 


\section{Analysis of the proposed approach}

In this section, first the error introduced by the beamspace transform is analyzed. Then, the CRB of the proposed approach is derived, which is more tight than the CRB derived in [34]. Finally, the computational complexity of the proposed approach is analyzed, and compared with that of the existing GMUSIC method.

\subsection{Analysis of error in the beamspace transform}

Due to the approximations in (12)-(14), the beamspace transform expounded in Section 3 inevitably introduces model transformation error, hence it is crucial to analyze the impact of this type of error on the performance of the proposed beamspace estimation approach. But the error introduced, i.e. $\varepsilon_{p}\left(\zeta_{k, j}(t), \theta_{k, j}(t)\right)$ in (11), has the asymptotic property

$\varepsilon_{p}\left(\zeta_{k, j}(t), \theta_{k, j}(t)\right) \rightarrow 0 \quad$ as $N \rightarrow \infty$.

A detailed proof is given in Appendix B.

Remark 3. For the lth UCA, the error caused by ignoring the residual term $\varepsilon_{p}\left(\zeta_{k, j}(t), \theta_{k, j}(t)\right)$ and its partial derivatives in (12)-(14) tends to zero as $N \rightarrow \infty$. In other words, the error introduced by the beamspace transform tends to zero as the number of UCyA antennas tends to infinity.

\subsection{Approximate Cramér-Rao bound}

The approximate CRB derived in [34] is based on only one UCA, which is not a realistic bound for the scenario where the BS is equipped with $L$ UCAs. Hence, a more realistic CRB is derived here. For the proposed estimator, the approximate CRB concerning the covariance matrix of the error of the estimated signal parameter vector $\mathbf{u}$ is given by

$\mathbf{C}=\left(\mathbf{J}_{\mathbf{u}, \mathbf{u}}-\mathbf{J}_{\mathbf{u}, \mathbf{v}} \mathbf{J}_{\mathbf{v}, \mathbf{v}}^{-1} \mathbf{J}_{\mathbf{u}, \mathbf{v}}^{T}\right)^{-1} \in \mathbb{R}^{4 K \times 4 K}$,

Table 1

Parameters in the simulations.

\begin{tabular}{ll}
\hline Number of UCAs $L$ & 2 \\
Number of antennas in each UCA $N$ & 50 \\
Number of UTs $K$ & 6 \\
Nominal azimuth DOAs $\bar{\theta}_{k}$ & $10^{\circ}, 50^{\circ}, 80^{\circ}$, \\
& $140^{\circ}, 180^{\circ}, 270^{\circ}$ \\
Nominal elevation DOAs $\bar{\phi}_{k}$ & $30^{\circ}, 40^{\circ}, 50^{\circ}$, \\
& $60^{\circ}, 70^{\circ}, 80^{\circ}$ \\
Azimuth angular spreads $\sigma_{\theta_{k}}$ & $1^{\circ}$ \\
Elevation angular spreads $\sigma_{\phi_{k}}$ & $1^{\circ}$ \\
Distance between adjacent UCAs $d$ & $0.5 \lambda$ \\
Radius of each UCA $r$ & $2 \lambda$ \\
Number of multipaths from the $k$ th UT $N_{k}$ & 50 \\
Variances of the path gains $\sigma_{\gamma_{k}}^{2}$ & 1 \\
Variance of the received noise $\sigma_{\mathrm{n}}^{2}$ & 1 \\
Number of received snapshots $T$ & 500 \\
Search precision of azimuth DOA & $0.02^{\circ}$ \\
Number of simulation trials & 200 \\
\hline
\end{tabular}

The 2-D nominal DOAs $\bar{\theta}_{k}, \bar{\phi}_{k}$ are sorted in ascending order of $k$; the 2-D angular spreads $\sigma_{\theta_{k}}, \sigma_{\phi_{k}}$, the numbers of multipaths $N_{k}$, and the variances of the path gains $\sigma_{\gamma_{k}}^{2}$ are the same for all the UTs. which implies

$\mathbb{E}\left\{(\hat{\mathbf{u}}-\mathbf{u})(\hat{\mathbf{u}}-\mathbf{u})^{T}\right\} \geq \mathbf{C}$.

A detailed derivation of (44) and the definitions of the variables used in (44) and (45) are offered in Appendix C.

Remark 4. Because the received signal is approximated by the Taylor series expansion, we can only derive the approximate Fisher information matrix (FIM) and the approximate CRB. The approximate CRB derived is an important metric for evaluating the performance of the proposed estimator. More specifically, this metric is viable for demonstrating the relative advantage (rather than the absolute performance) of the proposed estimator over the existing benchmark schemes. In the simulation results of Section 6, the approximate CRB is plotted as a reference to show that the proposed estimator outperforms the estimator of 34 .

\subsection{Complexity analysis}

In this subsection, the computational complexity of the proposed approach is analyzed, and it is compared with that of the existing GMUSIC method of [34] and subspace based approach of [31]. It is shown that the computational complexity of the proposed approach is significantly lower than that of the GMUSIC method and subspace based approach.

In this paper, the notation $O(n)$ means that the computational complexity of the arithmetic operations entailed is linear in $n \in \mathbb{R}^{+}[44, \mathrm{p}$. 5]. The number of snapshots $T$ is fixed, and the computational complexity of the representative algorithm considered is compared in the asymptotic sense as $N \rightarrow \infty$. In addition, the number of UCAs $L$ is set to 2 in the comparison with the existing approach. The computational complexities of these approaches for other values of $L$ may be compared in a similar way.

The computational complexities of Step 1, Step 2 and Step 5 in Algorithm 1 are $O\left(P^{\prime} N T\right), O\left(P^{\prime 2} T\right)$, and $O\left(P^{\prime} 3+P^{\prime 2} K D\right)$, respectively, where $D$ is the search dimension for estimating the nominal azimuth DOA of a single UT. The complexity of other steps in Algorithm 1 is

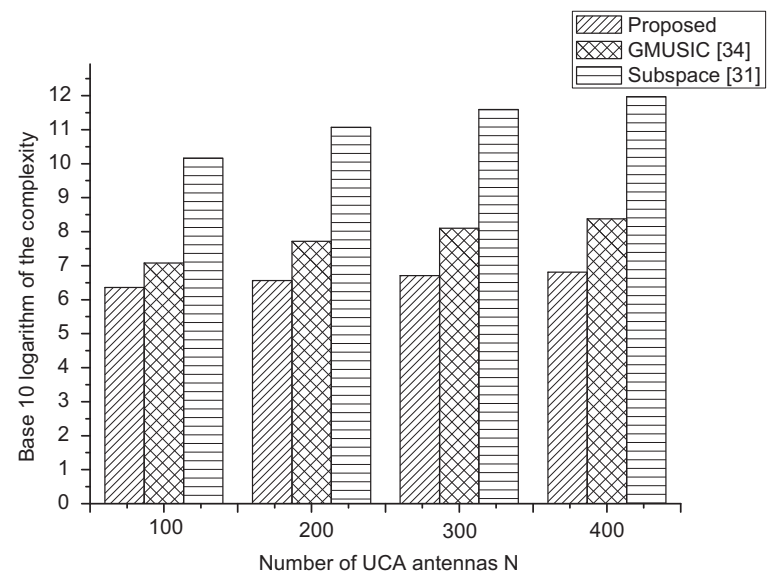

Fig. 3. Comparison of "the computational complexities of various estimation methods versus the number of UCA antennas $N$ " for the estimation of the angular parameters of six UTs. The $y$-axis represents the base 10 logarithm of the computational complexity in big $\mathrm{O}$ notation. 
a

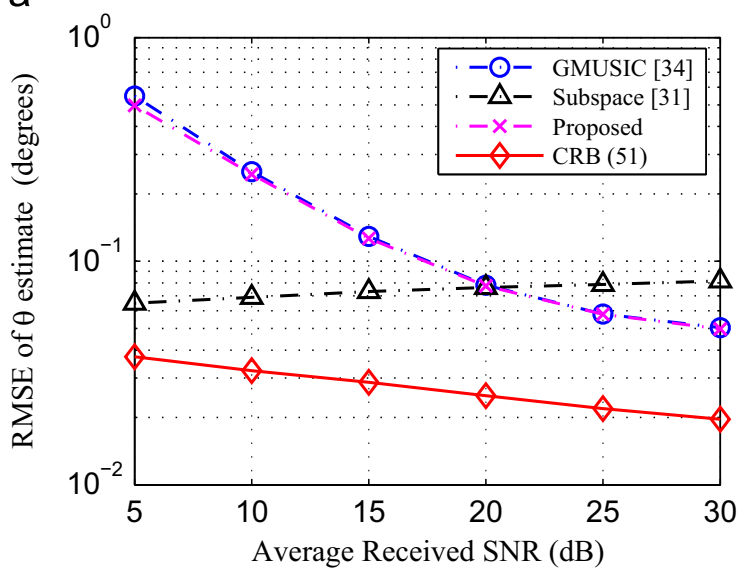

C

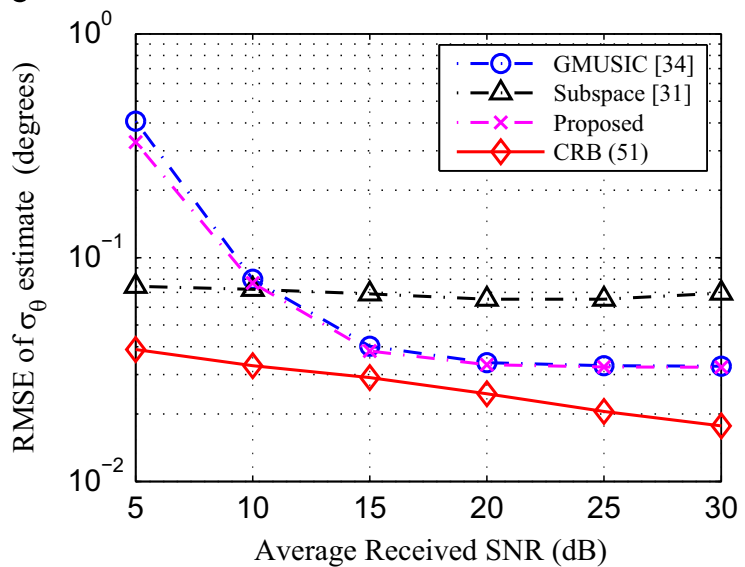

b

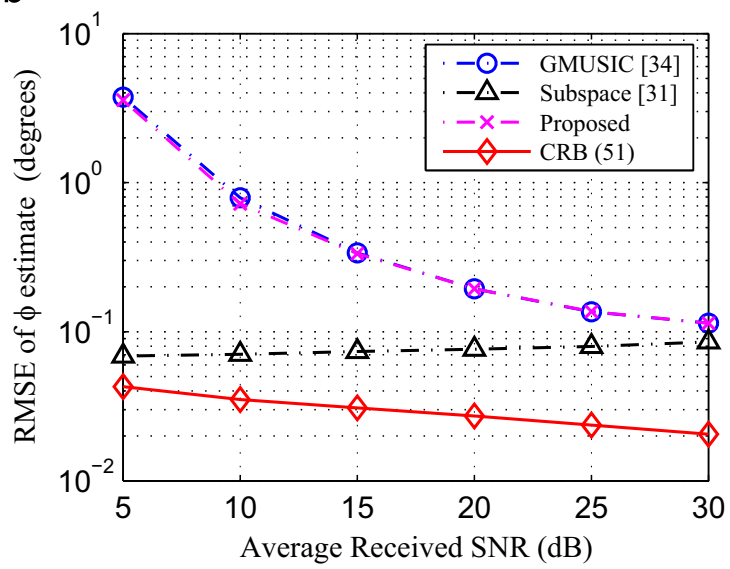

d

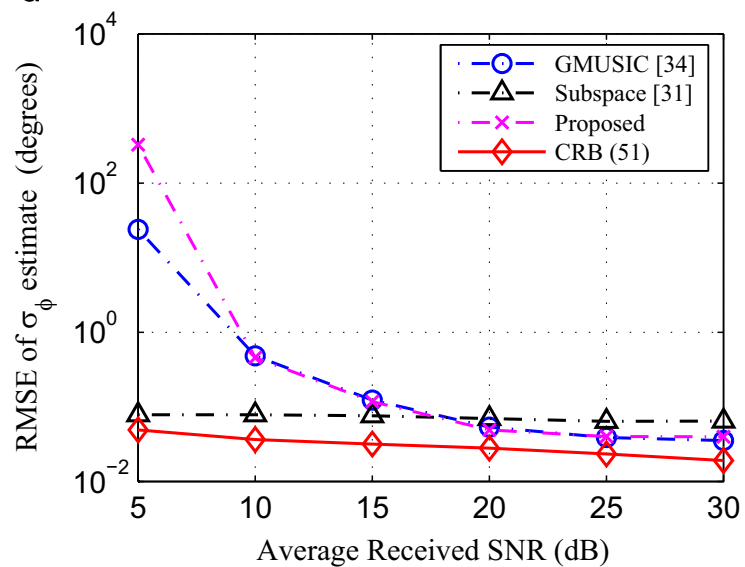

Fig. 4. Comparison of "RMSEs versus the average received SNR from each UT" for the estimation of different angular parameters of six UTs when using different estimation methods. (a), (b), (c), and (d) correspond to the estimation of the nominal azimuth DOA, the nominal elevation DOA, the azimuth angular spread, and the elevation angular spread, respectively.

$O\left(K^{3}+P^{\prime 3}\right)$. As a result, the computational complexity of the proposed approach is $O\left(N P^{\prime} T+P^{\prime 2} T+P^{\prime 3}+K^{3}+\right.$ $\left.P^{\prime 2} K D\right) \rightarrow O\left(N P^{\prime} T\right)$ as $N \rightarrow \infty$. The computational complexity of the GMUSIC approach of [34] can be calculated in a similar manner, which is $O\left(N^{2} T+N^{3}+K^{3}+N^{2} K D\right) \rightarrow O\left(N^{3}\right)$ as $N \rightarrow \infty$. Moreover, the computational complexity of the subspace based approach of [31] is $O\left(N^{2} T+N^{3} D^{\prime}\right) \rightarrow O\left(N^{3} D^{\prime}\right)$ as $N \rightarrow \infty$, where $D^{\prime}$ is the search dimension for estimating the nominal DOAs and the angular spreads of a single UT, and $D^{\prime} \gg D$.

From the above analysis, we can observe that the computational complexity of the proposed approach is remarkably lower than that of the existing GMUSIC and subspace based methods for large $N$.

\section{Numerical results}

In this section, numerical results are presented to show the performance and computational complexity of the proposed approach, the GMUSIC approach of [34], and the subspace based approach of [31]. Note that the subspace based approach of [31] is expected to exhibit better performance than these lower computational complexity approaches, such as the proposed approach and the GMUSIC approach. In addition, the performance is compared with the approximate CRB.

The parameters of the system are given in Table 1 . Note that some of the parameters, such as the numbers of multipaths $N_{k}$, are set the same for all users in most of our simulations just for simplicity. However, the scenario that each user has a different $N_{k}$ is also evaluated in what follows. The dimension of the beamspace received signal vector of each UCA, $P^{\prime}$, is chosen to be the larger value between $\lceil 2 \mu r+2\rceil$ and $3 K+1$. The transmitted signals $s_{k}(t), k=1,2, \ldots, K$, are BPSK modulated with covariances $\sigma_{s_{k}}^{2}$. It can be seen that the average received signal-to-noise ratio (SNR) of each BS antenna from the $k$ th UT is $\sigma_{s_{k}}^{2}$. The search range of the nominal azimuth DOA of the $k$ th UT is $\left[\bar{\theta}_{k}-0.98^{\circ}, \bar{\theta}_{k}+1^{\circ}\right]$. The search is restricted in this range, because the peak of the spectrum, cf. (37), cannot reach the maximum by taking values out of this range. For the subspace based approach of [31], the search range of the nominal DOA is $\left[\theta-0.1^{\circ}, \theta+0.1^{\circ}\right]$, where $\theta$ is the nominal DOA; and the search range of the angular spread is $\left[\sigma_{\theta}-0.1^{\circ}, \sigma_{\theta}+0.1^{\circ}\right]$, where $\sigma_{\theta}$ is the angular spread. The 
a
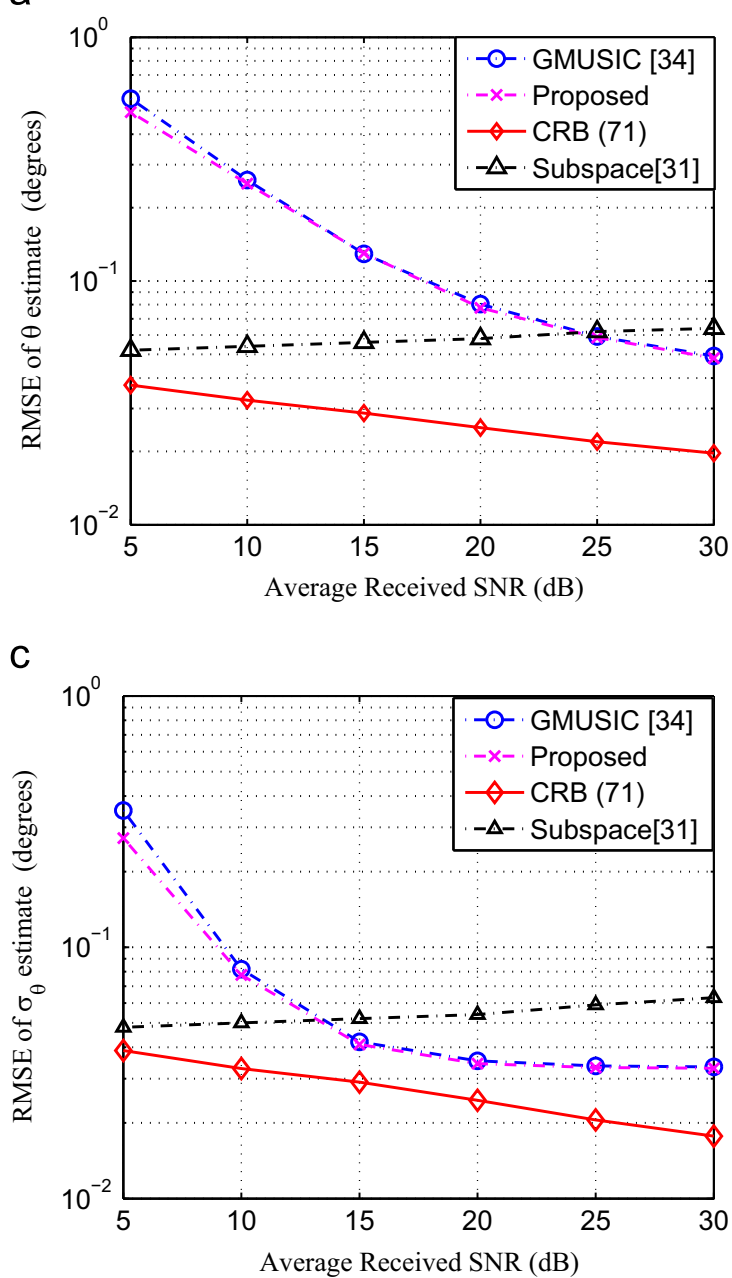

b

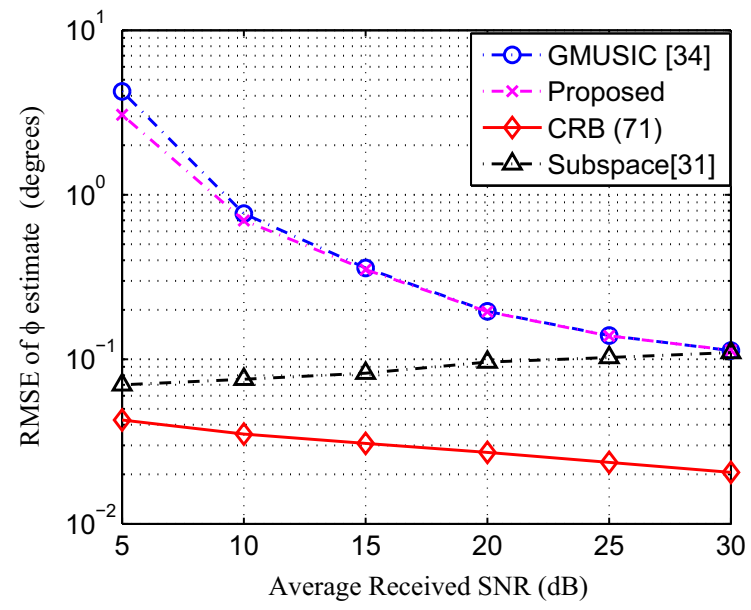

d

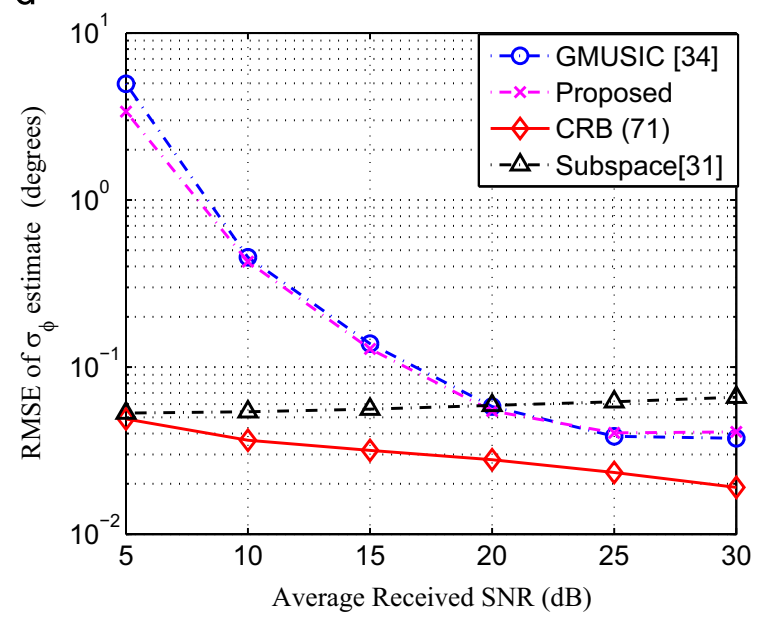

Fig. 5. Comparison of "RMSEs versus the average received SNR from each UT" for the estimation of different angular parameters of six UTs when using different estimation methods. The numbers of multipaths are random variables. (a), (b), (c), and (d) correspond to the estimation of the nominal azimuth DOA, the nominal elevation DOA, the azimuth angular spread, and the elevation angular spread, respectively.

performance of these estimators is evaluated by the metric of root mean square error (RMSE), which is averaged over all the trials and all the UTs.

Subject to these estimation parameters, the computational complexities of the estimation approaches analyzed in Section 5.3 can be compared explicitly. $D=$ $(1-(-0.98)) / 0.02+1=10^{2}$ is the number of searched angles for estimating the nominal azimuth DOA. $D^{\prime}=11^{4}=1.4641 \times 10^{4}$ is the search dimension of the angular parameters of one UT in the subspace based approach, where $11=(0.1-(-0.1)) / 0.02+1$ corresponds to the search of the angular parameter. Meanwhile, we have $\lceil 2 \mu r+2\rceil=28$ and $3 K+1=19$, thus the dimension of the beamspace received signal vector of each UCA is $P^{\prime}=28$. When $N=100$, the computational complexity of the proposed approach is $O\left(N P^{\prime} T+P^{\prime 2} T+P^{\prime 3}+K^{3}+P^{\prime 2} K D\right.$ )$=O\left(2.3 \times 10^{6}\right)$, the computational complexity of the GMUSIC approach in [34] is $O\left(N^{2} T+N^{3}+K^{3}+N^{2} K D\right)$ $=O\left(1.2 \times 10^{7}\right)$, and the computational complexity of the subspace based approach in [31] is $O\left(N^{2} T+N^{3} D^{\prime}\right)$
$=O\left(1.4 \times 10^{10}\right)$. Obviously, the computational complexity of the proposed approach is significantly lower than that of the methods of [34,31]. Therefore, the proposed approach is more favorable for implementation in massive MIMO systems. In Fig. 3, the computational complexity versus the number of UCA antennas is shown. It is clear that the proposed approach is of much lower computational complexity than the existing GMUSIC approach and subspace based approach for large UCAs. Since the computational complexity of the subspace based approach in [31] is very high, we only compare the proposed approach with this approach in Fig. 4, which shows the performance penalty of the proposed approach relative to the subspace based approach of [31].

In Fig. 4, RMSEs of the estimated nominal DOAs and angular spreads versus the average received SNR from each UT are shown. This figure shows that the RMSEs of the proposed approach are close to that of the GMUSIC in [34]. The RMSEs of the subspace based approach in [31] are almost invariant with the SNR. This is because the subspace 
a

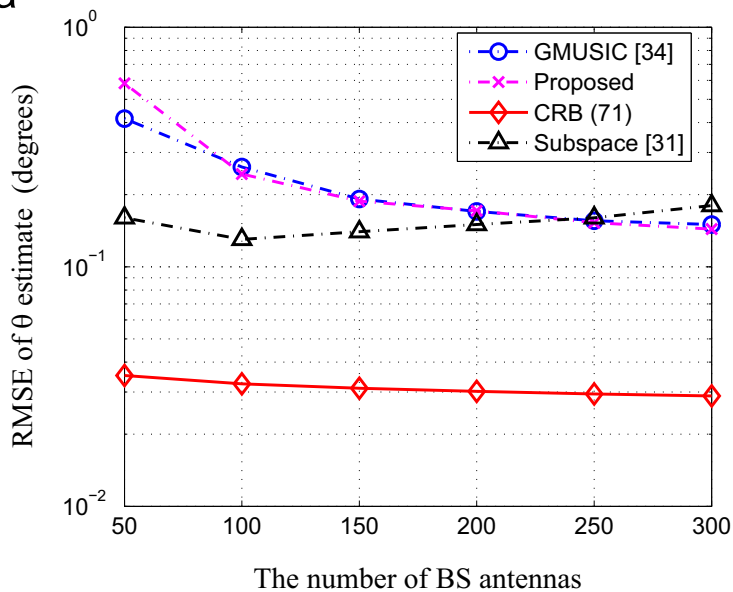

C

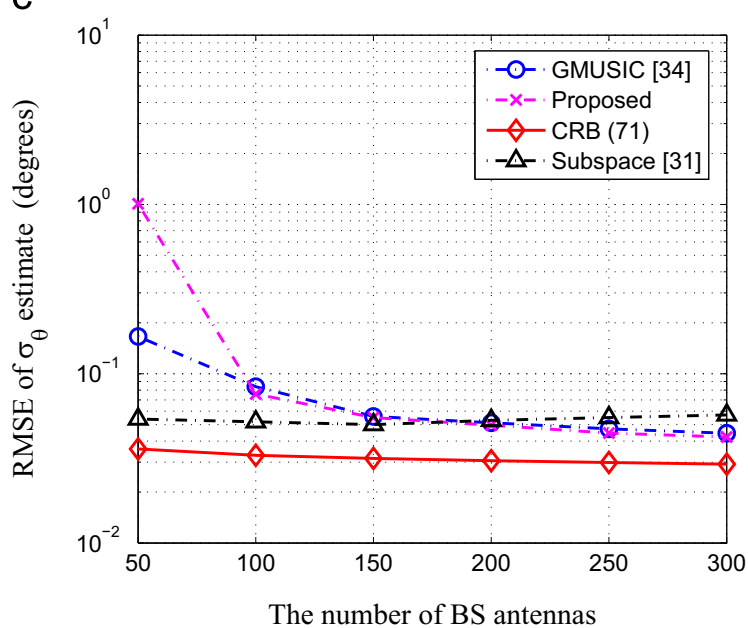

b

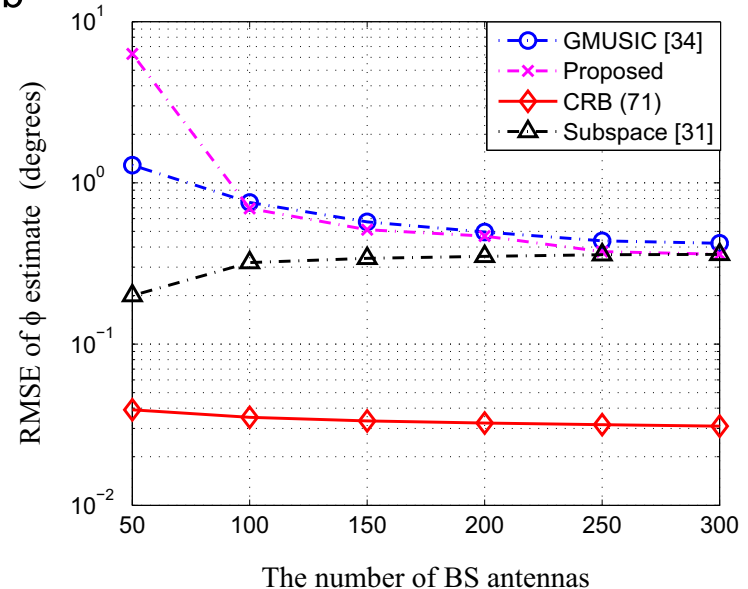

d

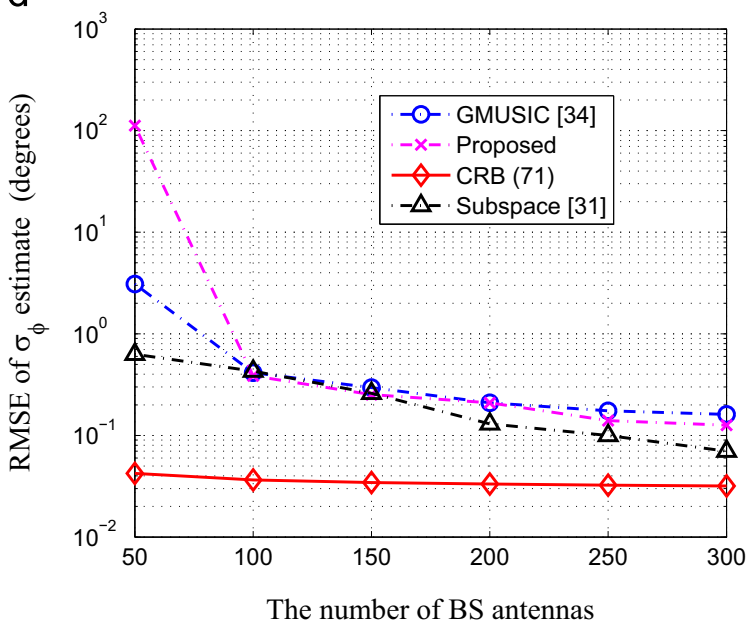

Fig. 6. Comparison of "RMSEs versus the number of BS antennas M" for the estimation of different angular parameters of six UTs when using different estimation methods. (a), (b), (c), and (d) correspond to the estimation of the nominal azimuth DOA, the nominal elevation DOA, the azimuth angular spread, and the elevation angular spread, respectively.

based approach does not need high SNR to achieve its best performance. This result coincides with the simulation result in [31]. For the nominal azimuth DOA and the angular spreads, the RMSEs of the proposed approach are smaller than that of the subspace based approach in the high SNR region. For the nominal elevation DOA, the RMSE of the proposed approach gets close to that of the subspace based approach as the SNR increases. In addition, the RMSEs of the angular spread estimates of the proposed approach get close to the approximate CRB as the SNR increases. These results demonstrate that the proposed approach performs better than the approach of [31] and performs close to the approach of [34] in massive MIMO systems.

In Fig. 5, we evaluate the RMSEs versus the average received SNR from each UT when assuming that each UT has a different number of multipaths. The number of multipaths of the $k$ th user, $N_{k}$, is an independent random variable in the interval $[1,100]$. We can see that the RMSEs of the DOA parameters estimated by the proposed approach and the GMUSIC approach of [34] decrease when the SNR increases, while the RMSEs resulted by using the subspace based approach of [31] slowly increase. These results demonstrate that if each UT has a different number of multipaths, the proposed approach exhibits a better performance than that of [31] when the SNR is high, and it performs close to that of [34] in the entire SNR region considered.

In Fig. 6, RMSEs of the estimated nominal DOAs and angular spreads versus the number of UCA antennas are shown, where the average received SNR from each UT is set to $10 \mathrm{~dB}$. We observe that the RMSE performance of the proposed approach is worse than that of [34,31] when the number of UCA antennas is less than 100. However, the RMSEs of the proposed approach decrease faster than that of [34] as the number of UCA antennas increases, and become lower than that of [34] when the number of UCA antennas is larger than 100. Meanwhile, the RMSEs of these DOA parameters estimated by the subspace based approach of [31] are almost invariant when the number of UCA antennas increases, which implies that the subspace based approach has roughly its best possible performance when the number of UCA antennas is not large. These results indicate that the estimation error caused by ignoring the 
a

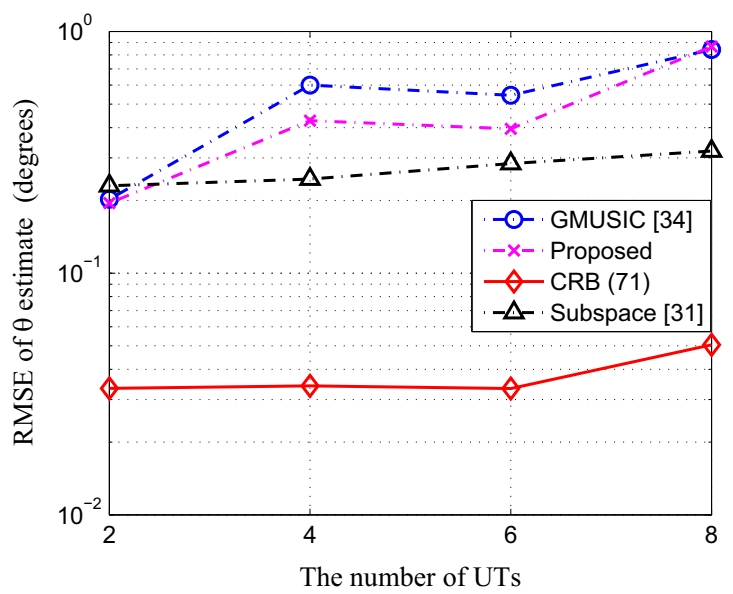

C

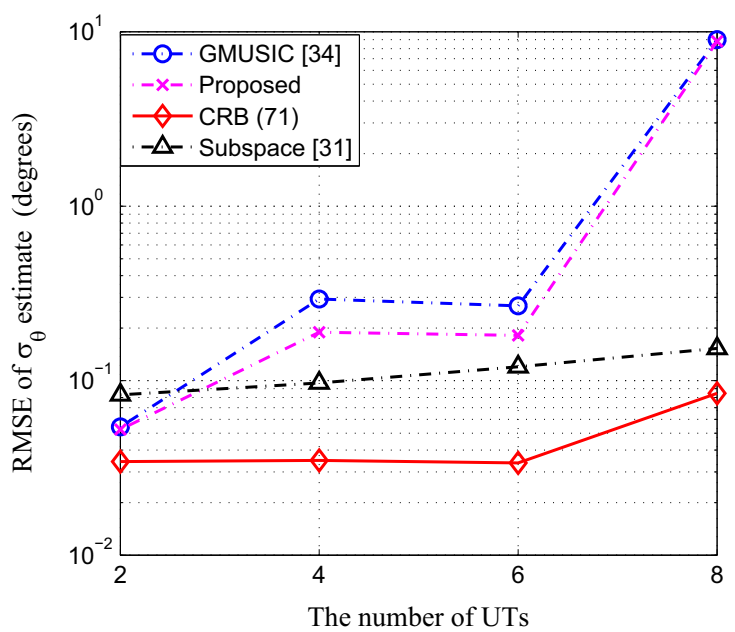

b

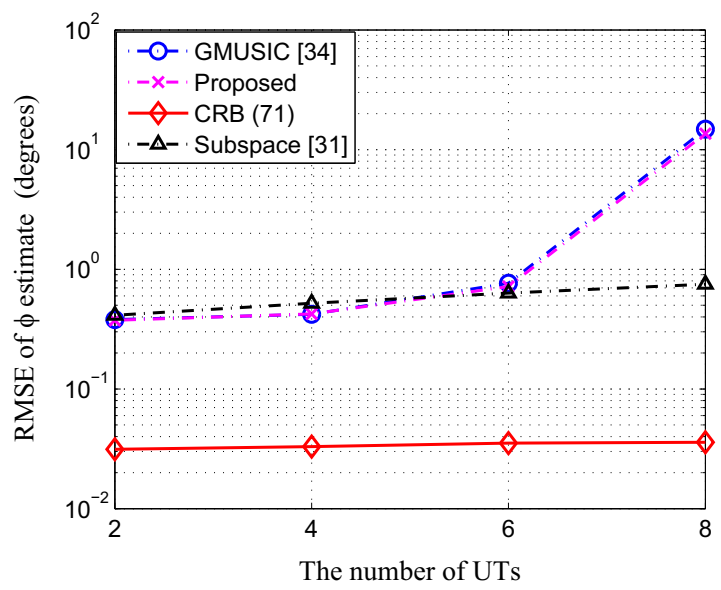

d

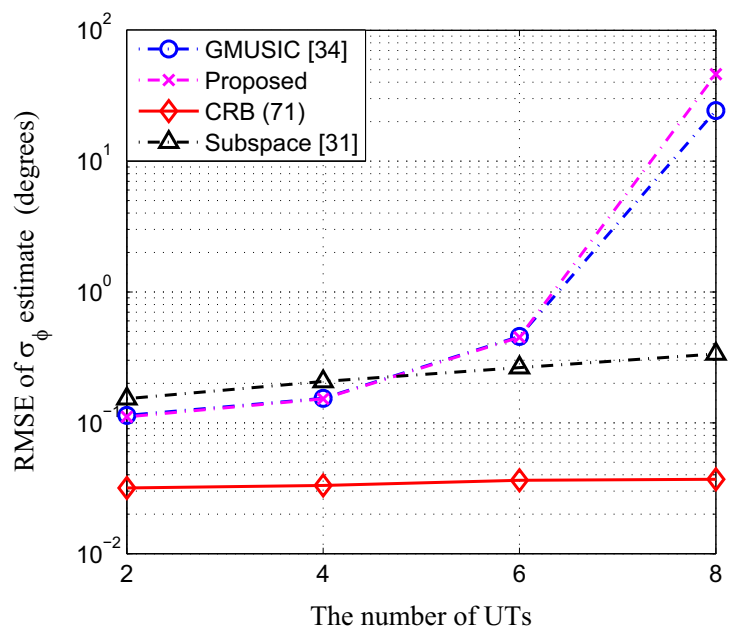

Fig. 7. Comparison of "RMSEs versus the number of UTs" for the estimation of different angular parameters of multiple UTs when using different estimation methods. (a), (b), (c), and (d) correspond to the estimation of the nominal azimuth DOA, the nominal elevation DOA, the azimuth angular spread, and the elevation angular spread, respectively.

residual term in (11) is large when the number of UCA antennas is small, and this estimation error decreases as the number of UCA antennas increases, which in turn improves the performance of the proposed approach. As a result, the proposed approach tends to outperform that of [34,31] as the number of UCA antennas increases. It is widely expected that the number of antennas can be a hundred or a few hundred in LSASs $[45,46]$. Thus, the proposed approach is more attractive than the GMUSIC and Subspace approach in future massive MIMO systems.

In Fig. 7, RMSEs of the estimated nominal DOAs and angular spreads versus the number of UTs are shown. We assume that two more UTs send signals to the BS, thus there are eight UTs send signals to the BS. Additionally, the nominal azimuth DOAs are $\bar{\theta}_{7}=240^{\circ}, \bar{\theta}_{8}=270^{\circ}$, and the nominal elevation DOAs are $\bar{\phi}_{7}=10^{\circ}, \bar{\phi}_{8}=20^{\circ}$. From Fig. 7 we can see that the RMSEs of the proposed approach and of the GMUSIC approach of [34] increase when the number of UTs is increased. This is because increasing of the UTs augments of the remainder of the Taylor series in (21).
As a result, the performance of the proposed approach degrades as the number of the UTs increases. In contrast, the RMSEs of the subspace based approach of [31] increase slowly when the number of the UTs is increased since the nominal DOAs of the UTs are estimated by only searching around the true values in the subspace based approach. The extension of the proposed approach to support more UTs is our future work.

\section{Conclusions}

In this paper, a generalized low-complexity generalized beamspace approach is proposed for 2-D localization of ID sources in the context of massive MIMO systems that rely on systems which use a large-scale UCyA. The problem considered entails estimating nominal azimuth and elevation DOAs and the corresponding angular spreads of multiple UTs. As a beneficial result of transforming the received signal vectors from the element space into the beamspace, the 
dimensions of the received signal vectors are reduced. Additionally, for benchmarking purpose, the ESPRIT and MUSIC based methods are extended to the considered scenario of the 2-D localization of ID sources. Compared with the generalized MUSIC approach which is the simplest among the traditional methods, the proposed approach not only has better performance, though marginally, but also imposes significantly lower computational complexity up to several orders of magnitude in the considered massive MIMO systems. Only small angular spread is considered in our paper. The extension of the proposed approach to the scenario where sources having large angular spreads may be addressed in our future work.

\section{Acknowledgment}

This work was supported in part by the National Natural Science Foundation of China under Grant 61271188, and Grant 61401041; by the National High Technology Research and Development Program of China (863 Program) under Grant 2015AA01A706 and by the Fundamental Research Funds for the Central Universities under Grant 2014RC0106.

\section{Appendix A}

Proof of the Proposition 1. By substituting (12) into (18) and replacing $\theta_{k, j}(t), \phi_{k, j}(t)$ with $\bar{\theta}_{k}, \bar{\phi}_{k}$, we obtain

$\left[\mathbf{b}_{l}\left(\bar{\theta}_{k}, \bar{\phi}_{k}\right)\right]_{p+P+1}=\alpha_{p, k} \exp \left(-i \mu d(l-1) \cos \left(\bar{\phi}_{k}\right)\right)$,

where $\alpha_{p, k}=\sqrt{N} J_{p}\left(\zeta_{k}\right) \exp \left(i p \bar{\theta}_{k}\right)$ and $\zeta_{k}=\mu r \sin \left(\bar{\phi}_{k}\right)$. Obviously, the following linear recurrence relation:

$\mathbf{b}_{l^{\prime}+1}\left(\bar{\theta}_{k}, \bar{\phi}_{k}\right)=\mathbf{b}_{l^{\prime}}\left(\bar{\theta}_{k}, \bar{\phi}_{k}\right) \exp \left(-i \mu d \cos \left(\bar{\phi}_{k}\right)\right)$

holds true for $l^{\prime}=1,2, \ldots, L-1$. Similarly, from (13) and (18), we have

$\frac{\partial\left[\mathbf{b}_{l}\left(\bar{\theta}_{k}, \bar{\phi}_{k}\right)\right]_{p+P+1}}{\partial \bar{\theta}_{k}}=i p \alpha_{p, k} \exp \left(-i \mu d(l-1) \cos \left(\bar{\phi}_{k}\right)\right)$

and the linear recurrence relation

$\frac{\partial \mathbf{b}_{l^{\prime}+1}\left(\bar{\theta}_{k}, \bar{\phi}_{k}\right)}{\partial \bar{\theta}_{k}}=\frac{\partial \mathbf{b}_{l^{\prime}}\left(\bar{\theta}_{k}, \bar{\phi}_{k}\right)}{\partial \bar{\theta}_{k}} \exp \left(-i \mu d \cos \left(\bar{\phi}_{k}\right)\right)$.

Furthermore, similar to the derivation of (46), from (14) and (18), we obtain the relation

$$
\begin{aligned}
& \frac{\partial\left[\mathbf{b}_{l}\left(\bar{\theta}_{k}, \bar{\phi}_{k}\right)\right]_{p+P+1}}{\partial \bar{\phi}_{k}}=\beta_{p, k} \exp \left(-i \mu d(l-1) \cos \left(\bar{\phi}_{k}\right)\right) \\
& \quad+i \mu d \alpha_{p, k} \sin \left(\bar{\phi}_{k}\right)(l-1) \exp \left(-i \mu d(l-1) \cos \left(\bar{\phi}_{k}\right)\right),
\end{aligned}
$$

where

$\beta_{p, k}=\sqrt{N}\left(J_{p-1}\left(\zeta_{k}\right)-J_{p+1}\left(\zeta_{k}\right)\right) \exp \left(i p \bar{\theta}_{k}\right)\left(\frac{1}{2} \mu r \cos \left(\bar{\phi}_{k}\right)\right)$.

Consequently, the linear relation

$$
\begin{aligned}
\frac{\partial \mathbf{b}_{l^{\prime}+1}\left(\bar{\theta}_{k}, \bar{\phi}_{k}\right)}{\partial \bar{\phi}_{k}}= & \frac{\partial \mathbf{b}_{l^{\prime}}\left(\bar{\theta}_{k}, \bar{\phi}_{k}\right)}{\partial \bar{\phi}_{k}} \exp \left(-i \mu d \cos \left(\bar{\phi}_{k}\right)\right) \\
& +i \mu d \sin \left(\bar{\phi}_{k}\right) \alpha_{p, k} \exp \left(-i \mu d l^{\prime} \cos \left(\bar{\phi}_{k}\right)\right)
\end{aligned}
$$

is established. Substituting (46) into (51), we obtain

$$
\begin{aligned}
\frac{\partial \mathbf{b}_{l^{\prime}+1}\left(\bar{\theta}_{k}, \bar{\phi}_{k}\right)}{\partial \bar{\phi}_{k}}= & \left(\frac{\partial \mathbf{b}_{l^{\prime}}\left(\bar{\theta}_{k}, \bar{\phi}_{k}\right)}{\partial \bar{\phi}_{k}}+\mathbf{b}_{l^{\prime}}\left(\bar{\theta}_{k}, \bar{\phi}_{k}\right) i \mu d \sin \left(\bar{\phi}_{k}\right)\right) \\
& \times \exp \left(-i \mu d \cos \left(\bar{\phi}_{k}\right)\right) .
\end{aligned}
$$

For the sake of conciseness, the linear recurrence relations in (47), (49) and (52) can be reformulated into the matrix form of Proposition 1.

\section{Appendix B. Proof of the asymptotic property}

Substituting (2), (8), and (9), into (10), we have

$$
\begin{aligned}
f_{p, l}\left(\theta_{k, j}(t), \phi_{k, j}(t)\right)= & \frac{1}{N} \sum_{n=1}^{N} \exp \left(i p \vartheta_{n}\right) \\
& \times \exp \left(i \mu r \sin \left(\phi_{k, j}(t)\right) \cos \left(\theta_{k, j}(t)-\vartheta_{n}\right)\right) \\
& \times \exp \left(-i \mu d(l-1) \cos \left(\phi_{k, j}(t)\right)\right) .
\end{aligned}
$$

When the number of antennas in each UCA tends to infinity, i.e., $N \rightarrow \infty$, (53) is reformulated as

$$
\begin{aligned}
f_{p, l}\left(\theta_{k, j}(t), \phi_{k, j}(t)\right) \rightarrow & \frac{1}{2 \pi} \int_{0}^{2 \pi} \exp (i p \vartheta) \\
& \times \exp \left(i \mu r \sin \left(\phi_{k, j}(t)\right) \cos \left(\theta_{k, j}(t)-\vartheta\right)\right) \\
& \times \exp \left(-i \mu d(l-1) \cos \left(\phi_{k, j}(t)\right)\right) \mathrm{d} \vartheta \\
= & i^{p} J_{p}\left(\zeta_{k, j}(t)\right) \exp \left(i p \theta_{k, j}(t)\right) \\
& \times \exp \left(-i \mu d(l-1) \cos \left(\phi_{k, j}(t)\right)\right) .
\end{aligned}
$$

Comparing (11) with (54), we get the asymptotic property $\varepsilon_{p}\left(\zeta_{k, j}(t), \theta_{k, j}(t)\right) \rightarrow 0$ as $N \rightarrow \infty$.

\section{Appendix C. Derivation of the approximate CRB}

First, the array manifold, cf. (2), for $\theta_{k, j}(t)$ and $\phi_{k, j}(t)$, is approximated by

$$
\begin{aligned}
{\left[\mathbf{a}\left(\theta_{k, j}(t), \phi_{k, j}(t)\right]_{m} \approx\right.} & \exp \left(i \mu \left[r \sin \left(\bar{\phi}_{k}\right) \cos \left(\bar{\theta}_{k}-\vartheta_{n}\right)\right.\right. \\
& \left.\left.-d(l-1) \cos \left(\bar{\phi}_{k}\right)\right]\right) \\
& \times \exp \left(i _ { \mu } \tilde { \phi } _ { k , j } ( t ) \left[r \cos \left(\bar{\phi}_{k}\right) \cos \left(\bar{\theta}_{k}-\vartheta_{n}\right)\right.\right. \\
& \left.\left.+d(l-1) \sin \left(\bar{\phi}_{k}\right)\right]\right) \\
& \times \exp \left(i_{\mu} \tilde{\theta}_{k, j}(t)\left[-r \sin \left(\bar{\phi}_{k}\right) \sin \left(\bar{\theta}_{k}-\vartheta_{n}\right)\right]\right),
\end{aligned}
$$

where $m, n, l$ are defined in (2). This approximation is similar to that of [27]. According to (55), the covariance matrix $\mathbf{R}_{\mathbf{x}}$ of the received signal vector $\mathbf{x}(t)$ in (1) is given by

$\mathbf{R}_{\mathbf{x}} \approx \mathbb{E}\left\{\mathbf{x}(t) \mathbf{x}^{H}(t)\right\}=\sum_{k=1}^{K} \sigma_{k}^{2} \Xi_{k}+\sigma_{\mathrm{n}}^{2} \mathbf{I}_{M}$,

where $\sigma_{k}^{2}=\sigma_{s_{k}}^{2} \sigma_{\gamma_{k}}^{2}$. Furthermore, $\Xi_{k}$ can be written as

$\Xi_{k}=\left(\mathbf{a}\left(\bar{\theta}_{k}, \bar{\phi}_{k}\right) \mathbf{a}^{H}\left(\bar{\theta}_{k}, \bar{\phi}_{k}\right)\right) \odot \mathbf{B}_{k}=\mathbf{D}_{k} \mathbf{B}_{k} \mathbf{D}_{k}^{H}$,

where $\mathbf{D}_{k}=\operatorname{diag}\left(\mathbf{a}\left(\bar{\theta}_{k}, \bar{\phi}_{k}\right)\right) \in \mathbb{C}^{M \times M}$, and each entry of $\mathbf{B}_{k} \in \mathbb{R}^{M \times M}$ equals

$\left[\mathbf{B}_{k}\right]_{m_{1}, m_{2}}=\exp \left(-\frac{1}{2} \mu^{2}\left(\eta_{k, m_{1}, m_{2}}^{2}+\rho_{k, m_{1}, m_{2}}^{2}\right)\right)$, 
in which $\eta_{k, m_{1}, m_{2}}=\sigma_{\phi_{k}}\left[r \cos \left(\bar{\phi}_{k}\right)\left(\cos \left(\bar{\theta}_{k}-\vartheta_{n_{1}}\right)-\cos \right.\right.$ $\left.\left.\left(\bar{\theta}_{k}-\vartheta_{n_{2}}\right)\right)+d\left(l_{1}-l_{2}\right) \sin \left(\bar{\phi}_{k}\right)\right], \rho_{k, m_{1}, m_{2}}=\sigma_{\theta_{k}} r \sin \left(\bar{\phi}_{k}\right)\left(\sin \left(\bar{\theta}_{k}\right.\right.$ $\left.\left.-\vartheta_{n_{1}}\right)-\sin \left(\bar{\theta}_{k}-\vartheta_{n_{2}}\right)\right), \quad m_{1}=N\left(l_{1}-1\right)+n_{1}$, and $m_{2}=N\left(l_{2}\right.$ $-1)+n_{2}$. Here we have $n_{1}=1,2, \ldots, N, n_{2}=1,2, \ldots$ $, N, l_{1}=1,2, \ldots, L$, and $l_{2}=1,2, \ldots, L$.

In Section 2, we have already verified that the received signal $\mathbf{x}(t)$ is a zero-mean circularly symmetric complexvalued Gaussian vector. Typically, the FIM is required for deriving the CRB. Because the received signal is approximated with the aid of (55), we can only derive the approximate FIM and the approximate CRB. This explains why we introduced the term "approximate CRB". Let us define $\quad \mathbf{u}=\left[\mathbf{u}_{\bar{\theta}}^{T}, \mathbf{u}_{\bar{\phi}}^{T}, \mathbf{u}_{\sigma_{\theta}}^{T}, \mathbf{u}_{\sigma_{\phi}}^{T}\right]^{T} \in \mathbb{R}^{4 K \times 1}, \quad \mathbf{v}=\left[\sigma_{1}^{2}, \sigma_{2}^{2}, \ldots, \sigma_{K}^{2}\right.$, $\left.\sigma_{\mathrm{n}}^{2}\right]^{T} \in \mathbb{R}^{(K+1) \times 1}, \quad$ and $\boldsymbol{\xi}=\left[\mathbf{u}^{T}, \mathbf{v}^{T}\right]^{T} \in \mathbb{R}^{(5 K+1) \times 1}$, where $\mathbf{u}_{\bar{\theta}}=\left[\bar{\theta}_{1}, \bar{\theta}_{2}, \ldots, \bar{\theta}_{K}\right]^{T} \in \mathbb{R}^{K \times 1}, \quad \mathbf{u}_{\bar{\phi}}=\left[\bar{\phi}_{1}, \bar{\phi}_{2}, \ldots, \bar{\phi}_{K}\right]^{T} \in \mathbb{R}^{K \times 1}$, $\mathbf{u}_{\sigma_{\theta}}=\left[\sigma_{\theta_{1}}, \sigma_{\theta_{2}}, \ldots, \sigma_{\theta_{K}}\right]^{T} \in \mathbb{R}^{K \times 1}$, and $\mathbf{u}_{\sigma_{\phi}}=\left[\sigma_{\phi_{1}}, \sigma_{\phi_{2}}, \ldots, \sigma_{\phi_{K}}\right]^{T}$ $\in \mathbb{R}^{K \times 1}$, then the approximate (finite-sample) Fisher information matrix (FIM) $\mathbf{J}_{\xi, \xi} \in \mathbb{R}^{(5 K+1) \times(5 K+1)}$ is expressed as $[47$, p. 525]

$\left[\mathbf{J}_{\xi, \xi}\right]_{q, q^{\prime}}=T \operatorname{tr}\left(\mathbf{R}_{\mathbf{x}}^{-1} \frac{\partial \mathbf{R}_{\mathbf{x}}}{\partial[\xi]_{q}} \mathbf{R}_{\mathbf{x}}^{-1} \frac{\partial \mathbf{R}_{\mathbf{x}}}{\partial[\xi]_{q^{\prime}}}\right)$,

where $q=1,2, \ldots, 5 K+1, q^{\prime}=1,2, \ldots, 5 K+1$, and $T$ is the number of received signal snapshots. From (56) and (57), the following partial derivatives can be obtained, which are

$$
\begin{aligned}
& \frac{\partial \mathbf{R}_{\mathbf{x}}}{\partial \bar{\theta}_{k}} \approx \sigma_{k}^{2}\left(\mathbf{D}_{\bar{\theta}_{k}} \mathbf{D}_{k} \mathbf{B}_{k} \mathbf{D}_{k}^{H}-\mathbf{D}_{k} \mathbf{B}_{k} \mathbf{D}_{k}^{H} \mathbf{D}_{\bar{\theta}_{k}}+\mathbf{D}_{k}\left(\mathbf{B}_{k} \odot \mathbf{B}_{\bar{\theta}_{k}}\right) \mathbf{D}_{k}^{H}\right), \\
& \frac{\partial \mathbf{R}_{\mathbf{x}}}{\partial \bar{\phi}_{k}} \approx \sigma_{k}^{2}\left(\mathbf{D}_{\bar{\phi}_{k}} \mathbf{D}_{k} \mathbf{B}_{k} \mathbf{D}_{k}^{H}-\mathbf{D}_{k} \mathbf{B}_{k} \mathbf{D}_{k}^{H} \mathbf{D}_{\bar{\phi}_{k}}+\mathbf{D}_{k}\left(\mathbf{B}_{k} \odot \mathbf{B}_{\bar{\phi}_{k}}\right) \mathbf{D}_{k}^{H}\right), \\
& \frac{\partial \mathbf{R}_{\mathbf{x}}}{\partial \sigma_{\theta_{k}}} \approx \sigma_{k}^{2}\left(\mathbf{D}_{k}\left(\mathbf{B}_{k} \odot \mathbf{B}_{\sigma_{\theta}, k}\right) \mathbf{D}_{k}^{H}\right), \\
& \frac{\partial \mathbf{R}_{\mathbf{x}}}{\partial \sigma_{\phi_{k}}} \approx \sigma_{k}^{2}\left(\mathbf{D}_{k}\left(\mathbf{B}_{k} \odot \mathbf{B}_{\sigma_{\phi}, k}\right) \mathbf{D}_{k}^{H}\right), \\
& \frac{\partial \mathbf{R}_{\mathbf{x}}}{\partial \sigma_{k}^{2}} \approx \mathbf{D}_{k} \mathbf{B}_{k} \mathbf{D}_{k}^{H} \\
& \frac{\partial \mathbf{R}_{\mathbf{x}}}{\partial \sigma_{\mathrm{n}}^{2}} \approx \mathbf{I}_{M},
\end{aligned}
$$

where $\mathbf{D}_{\bar{\theta}_{k}} \in \mathbb{C}^{M \times M}, \mathbf{D}_{\bar{\phi}_{k}} \in \mathbb{C}^{M \times M}, \mathbf{B}_{\bar{\theta}_{k}} \in \mathbb{C}^{M \times M}, \mathbf{B}_{\bar{\phi}_{k}} \in \mathbb{C}^{M \times M}$, $\mathbf{B}_{\sigma_{\theta}, k} \in \mathbb{C}^{M \times M}$, and $\mathbf{B}_{\sigma_{\phi}, k} \in \mathbb{C}^{M \times M}$ are defined as

$\left[\mathbf{D}_{\bar{\theta}_{k}}\right]_{m, m}=-i \mu r \sin \left(\bar{\phi}_{k}\right) \sin \left(\bar{\theta}_{k}-\vartheta_{n}\right)$,

$\left[\mathbf{D}_{\bar{\phi}_{k}}\right]_{m, m}=i \mu\left[r \cos \left(\bar{\phi}_{k}\right) \cos \left(\bar{\theta}_{k}-\vartheta_{n}\right)+d(l-1) \sin \left(\bar{\phi}_{k}\right)\right]$,

$\left[\mathbf{B}_{\bar{\theta}_{k}}\right]_{m_{1}, m_{2}}=-\eta_{k, m_{1}, m_{2}} \mu^{2} \sigma_{\phi_{k}} r \cos \left(\bar{\phi}_{k}\right) \times\left(-\sin \left(\bar{\theta}_{k}-\vartheta_{n_{1}}\right)\right.$

$\left.+\sin \left(\bar{\theta}_{k}-\vartheta_{n_{2}}\right)\right)-\rho_{k, m_{1}, m_{2}} \mu^{2} \sigma_{\theta_{k}} r \sin \left(\bar{\phi}_{k}\right)$

$\left(\cos \left(\bar{\theta}_{k}-\vartheta_{n_{1}}\right)-\cos \left(\bar{\theta}_{k}-\vartheta_{n_{2}}\right)\right)$,

$\left[\mathbf{B}_{\bar{\phi}_{k}}\right]_{m_{1}, m_{2}}=-\eta_{k, m_{1}, m_{2}} \mu^{2} \sigma_{\phi_{k}} \times\left[-r \sin \left(\bar{\phi}_{k}\right)\left(\cos \left(\bar{\theta}_{k}-\vartheta_{n_{1}}\right)\right.\right.$

$\left.\left.-\cos \left(\bar{\theta}_{k}-\vartheta_{n_{2}}\right)\right)+d\left(l_{1}-l_{2}\right) \cos \left(\bar{\phi}_{k}\right)\right]$

$-\rho_{k, m_{1}, m_{2}} \mu^{2} \sigma_{\theta_{k}} r \cos \left(\bar{\phi}_{k}\right)$

$\times\left(\sin \left(\bar{\theta}_{k}-\vartheta_{n_{1}}\right)-\sin \left(\bar{\theta}_{k}-\vartheta_{n_{2}}\right)\right)$,

$\left[\mathbf{B}_{\sigma_{\theta}, k}\right]_{m_{1}, m_{2}}=-\frac{\mu^{2}}{\sigma_{\theta_{k}}} \rho_{k, m_{1}, m_{2}}^{2}$,

and

$\left[\mathbf{B}_{\sigma_{\phi}, k}\right]_{m_{1}, m_{2}}=-\frac{\mu^{2}}{\sigma_{\phi_{k}}} \eta_{k, m_{1}, m_{2}}^{2}$, respectively. Note that $\mathbf{D}_{\bar{\theta}_{k}}, \mathbf{D}_{\bar{\phi}_{k}}$ are diagonal matrices. Similar to (58), $\mathbf{J}_{\mathbf{u}, \mathbf{u}} \in \mathbb{R}^{4 K \times 4 K}, \mathbf{J}_{\mathbf{u}, \mathbf{v}} \in \mathbb{R}^{4 K \times(K+1)}$, and $\mathbf{J}_{\mathbf{v}, \mathbf{v}} \in$ $\mathbb{R}^{(K+1) \times(K+1)}$ can be defined, and they are related to $\mathbf{J}_{\xi, \xi}$ by

$\mathbf{J}_{\xi, \xi}=\left[\begin{array}{ll}\mathbf{J}_{\mathbf{u}, \mathbf{u}} & \mathbf{J}_{\mathbf{u}, \mathbf{v}} \\ \mathbf{J}_{\mathbf{u}, \mathbf{v}}^{T} & \mathbf{J}_{\mathbf{v}, \mathbf{v}}\end{array}\right]$.

Finally, invoking the simple block matrix inversion lemma [48], the CRB concerning the covariance matrix of the estimation error of the angular parameter vector $\mathbf{u}$ is obtained as (44) and (45).

\section{References}

[1] D. Gesbert, M. Kountouris, R.W. Heath, C.-B. Chae, T. Sälzer, Shifting the MIMO paradigm, IEEE Signal Process. Mag. 24 (September (5)) (2007) 36-46.

[2] F. Rusek, D. Persson, B.K. Lau, E.G. Larsson, T.L. Marzetta, O. Edfors F. Tufvesson, Scaling up MIMO: opportunities and challenges with very large arrays, IEEE Signal Process. Mag. 30 (January (1)) (2013) 40-60.

[3] T.L. Marzetta, Noncooperative cellular wireless with unlimited numbers of base station antennas, IEEE Trans. Wirel. Commun. 9 (November (11)) (2010) 3590-3600.

[4] O.N. Alrabadi, E. Tsakalaki, H. Huang, G.F. Pedersen, Beamforming via large and dense antenna arrays above a clutter, IEEE J. Sel. Areas Commun. 31 (February (2)) (2013) 314-325.

[5] J. Koppenborg, H. Halbauer, S. Saur, C. Hoek, 3D beamforming trials with an active antenna array, in: Proceedings of ITG Workshop Smart Antennas, Dresden, Germany, March 2012, pp. 110-114.

[6] H. Halbauer, S. Saur, J. Koppenborg, C. Hoek, 3D beamforming performance improvement for cellular networks, Bell Labs Tech. J. 18 (September (2)) (2013) 37-56.

[7] H. Krim, M. Viberg, Two decades of array signal processing research: the parametric approach, IEEE Signal Process. Mag. 13 (July (4)) (1996) 67-94

[8] Y.U. Lee, J. Choi, I. Song, S.R. Lee, Distributed source modeling and direction-of-arrival estimation techniques, IEEE Trans. Signal Process. 45 (April (4)) (1997) 960-969.

[9] D. Astély, B. Ottersten, The effects of local scattering on direction of arrival estimation with MUSIC, IEEE Trans. Signal Process. 47 (April (12)) (1999) 3220-3234.

[10] S. Valaee, B. Champagne, P. Kabal, Parametric localization of distributed sources, IEEE Trans. Signal Process. 43 (September (9)) (1995) 2144-2153.

[11] S. Shahbazpanahi, S. Valaee, M.H. Bastani, Distributed source localization using ESPRIT algorithm, IEEE Trans. Signal Process. 49 (October (10)) (2001) 2169-2178.

[12] J. Lee, I. Song, H. Kwon, S.R. Lee, Low-complexity estimation of 2-D DOA for coherently distributed sources, Signal Process. 83 (August (8)) (2003) 1789-1802.

[13] J. Lee, J. Joung, J.D. Kim, A method for the direction-of-arrival estimation of incoherently distributed sources, IEEE Trans. Veh. Technol. 57 (September (5)) (2008) 2885-2893.

[14] Z. Zheng, G. Li, Low-complexity estimation of DOA and angular spread for an incoherently distributed source, Wirel. Pers. Commun. 70 (January (4)) (2013) 1653-1663.

[15] M. Bengtsson, B. Ottersten, Low-complexity estimators for distributed sources, IEEE Trans. Signal Process. 48 (August (8)) (2000) 2185-2194.

[16] S. Shahbazpanahi, S. Valaee, A.B. Gershman, A covariance fitting approach to parametric localization of multiple incoherently distributed sources, IEEE Trans. Signal Process. 52 (March (3)) (2004) 592-600.

[17] T. Trump, B. Ottersten, Estimation of nominal direction of arrival and angular spread using an array of sensors, Signal Process. 50 (April (1) 2)) (1996) 57-69.

[18] B.T. Sieskul, An asymptotic maximum likelihood for joint estimation of nominal angles and angular spreads of multiple spatially distributed sources, IEEE Trans. Signal Process. 59 (March (3)) (2010) $1534-1538$

[19] O. Besson, F. Vincent, P. Stoica, Approximate maximum likelihood estimators for array processing in multiplicative noise 
environments, IEEE Trans. Signal Process. 48 (September (9)) (2000) 2506-2518.

[20] M. Ghogho, O. Besson, A. Swami, Estimation of direction of arrival of multiple scattered sources, IEEE Trans. Signal Process. 49 (November (11)) (2001) 2467-2480.

[21] A.B. Gershman, C.F. Mecklenbräuker, J.F. Böhme, Matrix fitting approach to direction of arrival estimation with imperfect spatial coherence of wavefronts, IEEE Trans. Signal Process. 45 (July (7)) (1997) 1894-1899.

[22] O. Besson, P. Stoica, Decoupled estimation of DOA and angular spread for a spatially distributed source, IEEE Trans. Signal Process. 48 (July (7)) (2000) 1872-1882.

[23] O. Besson, P. Stoica, A.B. Gershman, Simple and accurate direction of arrival estimator in the case of imperfect spatial coherence, IEEE Trans. Signal Process. 49 (July (4)) (2001) 730-737.

[24] A. Monakov, O. Besson, Direction finding for an extended target with possibly non-symmetric spatial spectrum, IEEE Trans. Signal Process. 52 (January (1)) (2004) 283-287.

[25] A. Zoubir, Y. Wang, P. Chargé, A modified COMET-EXIP method for estimating a scattered source, Signal Process. 86 (April (4)) (2006) $733-743$.

[26] B. Ottersten, P. Stoica, R. Roy, Covariance matching estimation techniques for array signal processing applications, Digit. Signal Process. 8 (July (3)) (1998) 185-210.

[27] H. Boujemâa, Extension of COMET algorithm to multiple diffuse source localization in azimuth and elevation, Eur. Trans. Telecommun. 16 (November/December (6)) (2005) 557-566.

[28] Y. Meng, P. Stoica, K.M. Wong, Estimation of the directions of arrival of spatially dispersed signals in array processing, IEE Proc. Radar Sonar Navig. 143 (February (1)) (1996) 1-9.

[29] M. Bengtsson, B. Ottersten, A generalization of weighted subspace fitting to full-rank models, IEEE Trans. Signal Process. 49 (May (5)) (2001) 1002-1012.

[30] M. Bengtsson, Antenna array signal processing for high rank models (Ph.D. dissertation), Signals, Sensors, Systems Department, Royal Institute of Technology, Stockholm, Sweden, 1999.

[31] A. Zoubir, Y. Wang, P. Chargé, Efficient subspace-based estimator for localization of multiple incoherently distributed sources, IEEE Trans. Signal Process. 56 (February (2)) (2008) 532-542.

[32] A. Hassanien, S. Shahbazpanahi, A.B. Gershman, A generalized Capon estimator for localization of multiple spread sources, IEEE Trans. Signal Process. 52 (January (1)) (2004) 280-283.

[33] J. Zhou, Z. Zheng, G. Li, Low-complexity estimation of the nominal azimuth and elevation for incoherently distributed sources, Wirel. Pers. Commun. 71 (August (3)) (2013) 1777-1793.

[34] X. Guo, Q. Wan, X. Shen, H. Dou, Low-complexity parameters estimator for multiple 2D domain incoherently distributed sources Turk. J. Elect. Eng. Comput. Sci. 19 (May (3)) (2011) 445-462.
[35] R.O. Schmidt, Multiple emitter location and signal parameter estimation, IEEE Trans. Antennas Propag. 34 (March (3)) (1986) 276-280.

[36] A. Paulraj, R. Roy, T. Kailath, A subspace rotation approach to signal parameter estimation, Proc. IEEE 74 (July (7)) (1986) 1044-1046.

[37] R. Roy, A. Paulraj, T. Kailath, ESPRIT-a subspace rotation approach to estimation of parameters of cisoids in noise, IEEE Trans. Acoust. Speech Signal Process. 34 (October (5)) (1986) 1340-1342.

[38] R. Roy, T. Kailath, ESPRIT-estimation of signal parameters via rotational invariance techniques, IEEE Trans. Acoust. Speech Signal Process. 37 (July (7)) (1989) 984-995.

[39] A. Hu, T. Lv, H. Gao, Z. Zhang, S. Yang, An ESPRIT-based approach for 2-D localization of incoherently distributed sources in massive MIMO systems, IEEE J. Sel. Top. Signal Process. (March (99)) (2014) 1.

[40] C.P. Mathews, M.D. Zoltowski, Eigenstructure techniques for 2-D angle estimation with uniform circular arrays, IEEE Trans. Signal Process. 42 (September (9)) (1994) 2395-2407.

[41] G. Xu, S.D. Silverstein, R.H. Roy, T. Kailath, Beamspace ESPRIT, IEEE Trans. Signal Process. 42 (February (2)) (1994) 349-356.

[42] J.A. Gansman, M.D. Zoltowski, J.V. Krogmeier, Multidimensional multirate DOA estimation in beamspace, IEEE Trans. Signal Process. 44 (November (11)) (1996) 2780-2792.

[43] D.E.N. Davies, In: A.W. Rudge, et al. (Eds.), The Handbook of Antenna Design, vol. 2, Peregrinus, London, UK, 1983 (Chapter 12).

[44] G.H. Golub, C.F.V. Loan (Eds.), Matrix Computations, 3rd ed. The Johns Hopkins University Press, Baltimore, MD, 1996.

[45] H.Q. Ngo, E.G. Larsson, T.L. Marzetta, Energy and spectral efficiency of very large multiuser MIMO systems, IEEE Trans. Commun. 61 (April (4)) (2013) 1436-1449.

[46] H.Q. Ngo, E.G. Larsson, T.L. Marzetta, Uplink power efficiency of multiuser MIMO with very large antenna arrays, In: Proceedings of 49th Annual Allerton Conference on Communication, Control, and Computing (Allerton '11), Monticello, IL, September 2011, pp. 12721279.

[47] S.M. Kay, Fundamentals of Statistical Signal Processing: Estimation Theory, Prentice-Hall, Englewood Cliffs, NJ, 1993.

[48] S.E. Jo, S.W. Kim, T.J. Park, Equally constrained affine projection algorithm, In: Proceedings of 38th Asilomar Conference on Signals, Systems and Computers, Pacific Grove, CA, November 2004, pp. 955-959.

[49] P. Zetterberg, Mobile cellular communications with base station antenna arrays: spectrum efficiency, algorithms and propagation models (Ph.D. dissertation), Royal Institute of Technology, Stockholm, Sweden, 1997.

[50] W.C.Y. Lee, Mobile Communications Design Fundamentals, 2nd ed. Wiley, New York, 1992.

[51] D. Astely, B. Ottersten, The effects of local scattering on direction of arrival estimation with MUSIC, IEEE Trans. Signal Process. 41 (July (12)) (2007) 3220-3234. 